\title{
Understanding Test Modalities of Tire Grip and Laboratory-Road Correlations with Modeling
}

\author{
Marzieh Salehi $^{1}$ (D) Jacques W. M. Noordermeer ${ }^{1} \cdot$ Louis A. E. M. Reuvekamp $^{1,2} \cdot$ Anke Blume $^{1}$
}

Received: 15 April 2021 / Accepted: 22 July 2021 / Published online: 14 August 2021

(c) The Author(s) 2021

\begin{abstract}
The present study is meant to obtain tribological insight into the interface of a rolling rubber wheel on a counter-surface disk based on the work of the previous study Salehi et al. (Tribol Lett 68(1):37, 2020), in which a new test method was developed to rapidly predict tire grip in a laboratory environment. A Laboratory Abrasion Tester (LAT100) was used and exploited as a tribometer. This opened a new cost- and time-effective horizon for tire material development in a laboratory environment rather than having to test tread compounds by building full-scale tires. The method was validated by a comprehensive study for six different tire tread compositions, by correlating the laboratory data for solid rubber wheels as LAT100 specimens with real tire results in two test modalities: lateral $(\alpha)$ and longitudinal $(\kappa)$ sweep tests on a dry road. It was demonstrated that the LAT100 can be exploited to simulate the $\alpha$-sweep tire tests, but not the $\kappa$-sweep. The dynamics and physics of a rolling rubber wheel on a counter-surface disk of the LAT100 test step-up are investigated utilizing the renowned physical "brush model" in comparison to full-scale tire tests. The type of test modality leads to different friction mechanisms in the contact patch even at similar test conditions. This is substantiated by recognizing the two regions: stationary and non-stationary, in the contact area which results in different friction components and mechanisms. The behavior of the rolling wheel in lateral and longitudinal movements at the same test conditions is comparable if the contributions of the mentioned regions in the contact area are similar.
\end{abstract}

Keywords Tire tribology $\cdot$ Tire grip $\cdot$ Rubber friction $\cdot$ LAT100 $\cdot$ Brush model $\cdot$ Solid rubber wheel

\section{Introduction}

Tire grip or traction is a concept that describes the grasp and interaction between the tire and the road to avoid vehicle skidding or sliding, crucial for safety. Proper tire grip provides a good level of handling which is a prerequisite for vehicle steering in various driving states such as cornering, braking, and accelerating. The tire grip is the result of the generated frictional forces in the aforementioned driving states which are created by tire slippage in the contact patch.

Marzieh Salehi

m.salehi@utwente.nl

$\triangle$ Jacques W. M. Noordermeer

j.w.m.noordermeer@utwente.nl

1 Department of Elastomer Technology and Engineering, University of Twente, P.O. Box 217, 7500 AE Enschede, The Netherlands

2 Apollo Tyres Global R\&D B.V., Colosseum 2, 7521PT Enschede, The Netherlands
When a vehicle brakes, accelerates, or corners, the tire tread elements in the contact area move a noticeable displacement in relation to the road. This has been elaborated in detail in our previous study [1]. Among all available laboratory tribometers suitable for measuring tire tread frictional properties, great interest exists towards measurements with solid rubber wheels [1-18]. The main advantage is the rolling movement of the rubber wheel sample which is analogous to a tire. The friction properties of rubber materials as the output of the laboratory devices are often employed as input data for tire modeling and simulations. Using a rolling wheel sample provides a more realistic input for tire modeling due to better surface cooling, compared to sliding body measurements with continuous contact to the counter-surface [18].

In the previous study [1], the Laboratory Abrasion Tester (LAT100)—despite its name-was re-designed and employed as a laboratory tribometer for prediction of tire friction using a solid rubber wheel sample known as the Grosch wheel. First, it was demonstrated that the LAT100 can be exploited particularly to simulate the 
lateral $\alpha$-sweep tire tests, despite not being the longitudinal $\kappa$-sweep. The correlations were acquired experimentally and verified with statistical analyses. Second, it was shown that there was no correlation between the two different test modalities of $\alpha$ - and $\kappa$-sweep data of the tire at the peak points of the friction curves for all six tread compounds, though the test was performed at the same test conditions. Because both test modalities were outdoor types and the friction behaviors were measured on full-scale tire interfaces with the same road asphalt surface, it was deduced that the comparison between the acquired tire data demonstrated the differences in the two tire test modalities.

The present study supports the previous work by creating more insight into the wheel rolling dynamics to understand the acquired correlations between the laboratory and the road by comprehending the involved friction phenomena and mechanisms in the contact patch. By utilizing the physical "brush model" for tires, the dynamics and physics of a rolling rubber wheel on a counter-surface disk of the LAT100 test set-up are highlighted. Therewith, the behavior of the rolling wheel in lateral and longitudinal, compared to real tire movements is investigated. The intricate differences between the $\alpha$ and $\kappa$ sweep test configurations are elaborated. With this approach, the previous conclusions become more concrete and answers to remaining questions are provided.

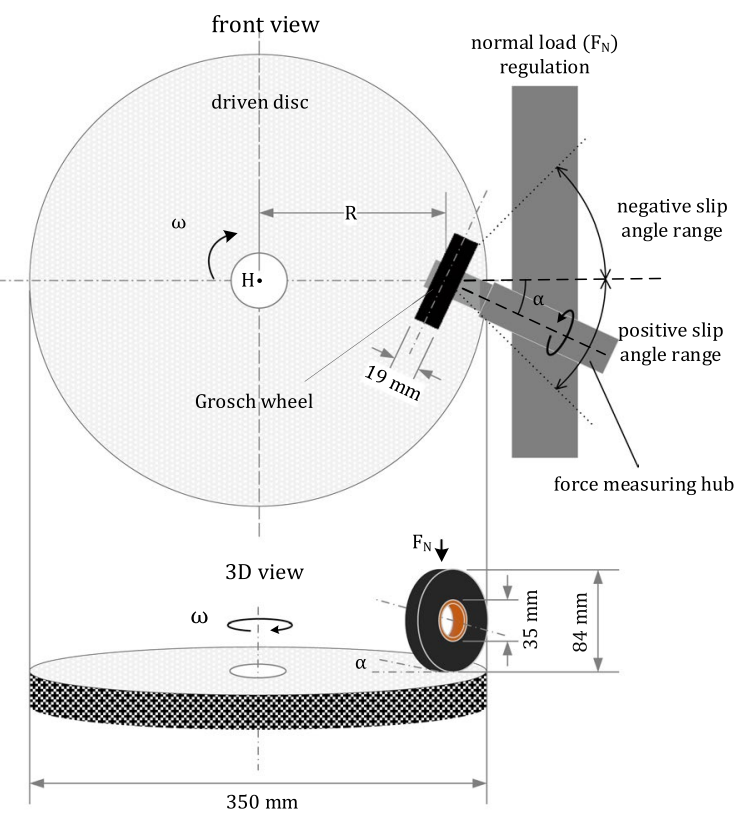

\section{The Physics and Dynamics of the LAT100 Device}

\subsection{Test Set-Up}

The LAT100 simulates tire-operating conditions to measure the frictional forces as a function of various slips, normal loads, and speeds on different counter-surface substrates. Figure 1 (left) shows a schematic view of the LAT100 test set-up. The machine consists of a driven disk with a diameter of $350 \mathrm{~mm}$ onto which a solid rubber wheel with outside and inside diameters of $84 \mathrm{~mm}$ and $35 \mathrm{~mm}$ and a thickness of $19 \mathrm{~mm}$, known as Grosch wheel, is pressed under a given normal load $F_{\mathrm{N}}(\mathrm{N})$ and run at a defined slip velocity of $V_{\mathrm{s}}$ $(\mathrm{km} / \mathrm{h})$. The slip velocity is created by combining the disk traveling velocity $V_{\mathrm{t}}$ and a slip angle $\alpha\left(^{\circ}\right)$. The basic principle is that the disk spinning with a certain traveling velocity induces rotation of the Grosch wheel with a circumferential velocity $V_{\mathrm{c}}$. Side (lateral) $F_{\mathrm{s}}$ and friction $F_{\mathrm{f}}$ forces are generated by the slip velocity and the normal force. Countersurface disks with various surface roughness can also be mounted. All three force components acting on the wheel during the tests are recorded with the load cell measuring hub. Figure 1 (Right) shows the trigonometry of the velocities and resultant forces of the Grosch wheel sample on the LAT100 disk at slip angle $\alpha$. The test set-up is arranged in a vertical alignment which eases the removal of abrasion debris. The frictional forces as direct outputs of the

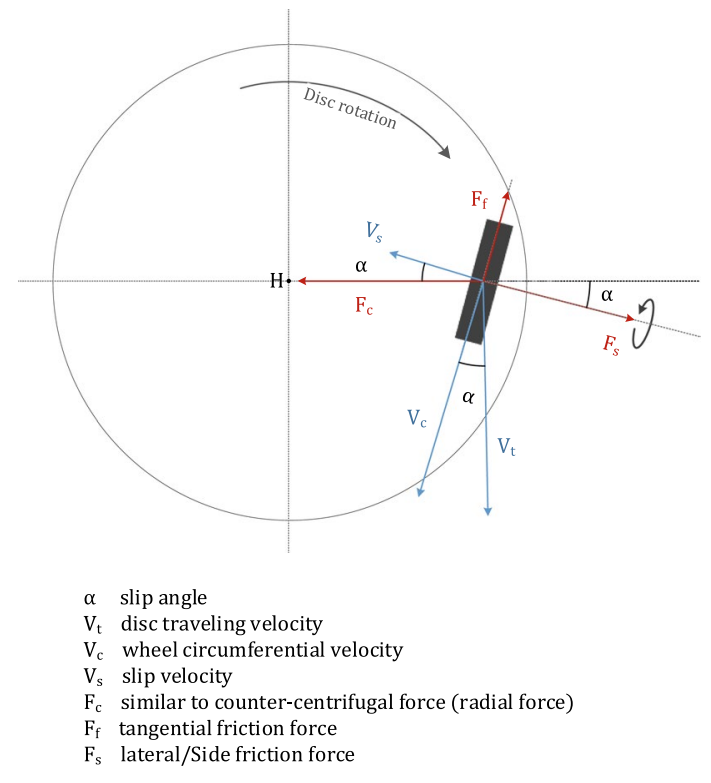

Fig. 1 (Left) Schematic view of the LAT100 test set-up, (Right) The trigonometry of velocities and the resultant forces of the Grosch wheel sample on the LAT100 counter-surface disk at slip angle $\alpha$ 
measurements with the LAT100 enable to rank and compare various rubber compounds.

\subsection{Curvature Effect of the Disk Counter-Surface}

Based on the gradient of the radial distance between the leading and trailing edge of the Grosch wheel to the disk center point $H$, the disk traveling velocity alters: $V_{\mathrm{t}}=r * \omega$, the further the point from the center, the larger the traveling velocity. Here, $R$ in Figs. 1 and 2 is the distance between the center of the contact area and the disk center point $H$, and $r$ varies around the $R$ value dependent on the slip angle and disk curvature. Consequently, the slip velocities vary; this is not the case if no curvature exists provided macro-scale speaking. Figure 2 depict a schematic view of the contact area of the Grosch wheel with and without a disk curvature at a slip angle $\alpha\left(^{\circ}\right)$.

The relationship between the traveling and slip velocities in the center point of the wheel contact with the countersurface disk is expressed in Eq. (1).

$V_{\mathrm{s}}=\mathrm{V}_{\mathrm{t}} \sin \alpha=R \omega \sin \alpha$.

At any other point in the contact area, the slip velocity varies according to Eq. (2).

$V_{\mathrm{s}}=V_{\mathrm{t}} \frac{r(x, y, \alpha)}{R} \sin \alpha$

where $r$ can be estimated as follows:

$r(x, y, \alpha)=R^{2}+x^{2}+y^{2}-2 R(y \cos \alpha+x \sin \alpha)$.

The derivation of $r$ is presented in "Appendix". On an actual road, the impact of a bend in cornering depends on the radius of the curvature which is most notable in sharp turns.

\subsection{Rubber Wheel Deflection}

A deflection $\delta$ for the rubber wheel-shaped sample occurs as shown in Fig. 3. As the normal load $F_{\mathrm{N}}$ increases, the

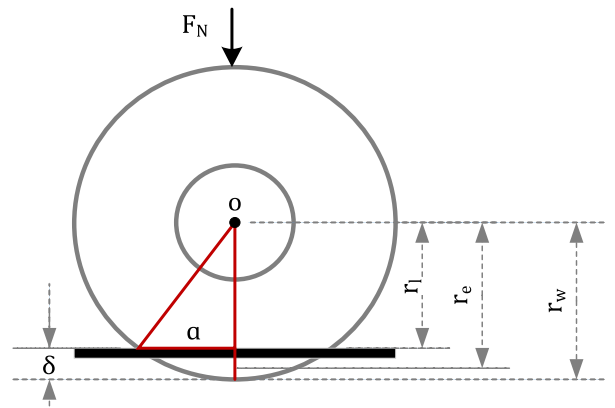

Fig. 3 Wheel deflection under normal load $F_{\mathrm{N}}$, vertical deflection $\delta$, loaded radius $r_{\mathrm{l}}$, effective radius $r_{\mathrm{e}}$, unloaded wheel radius $r_{\mathrm{w}}$, and $a$ is half of the contact length

wheel flattens more in the center of the contact area. The $r_{\mathrm{e}}$ is defined as the effective radius of the rubber wheel when spinning without an external torque applied to the rolling axis. Its value lies somewhere in between the unloaded radius $r_{\mathrm{w}}$ and the static loaded $r_{1}$ [19].

\section{Friction Modeling}

\subsection{Rolling Friction of a Tire}

The function of a tire on the road can only be achieved due to viscoelastic nature of a material called rubber. The tire is composed of various elements which are mainly rubber compounds, each contributing to the overall performance profile. It interfaces with the road which is also an intricate system. As a result, the tire is considered a non-linear function of a multitude of inputs and outputs such as velocity, longitudinal slip $\kappa$, side slip $\alpha$, inclination (camber) angle $\gamma$, and forces and moments in different directions. Above all, road conditions, temperature, tire inflation pressure, and wear have roles to play. To predict tire grip or friction, considering all contributing factors in one laboratory device seems an elaborate task to accomplish. However, the tread
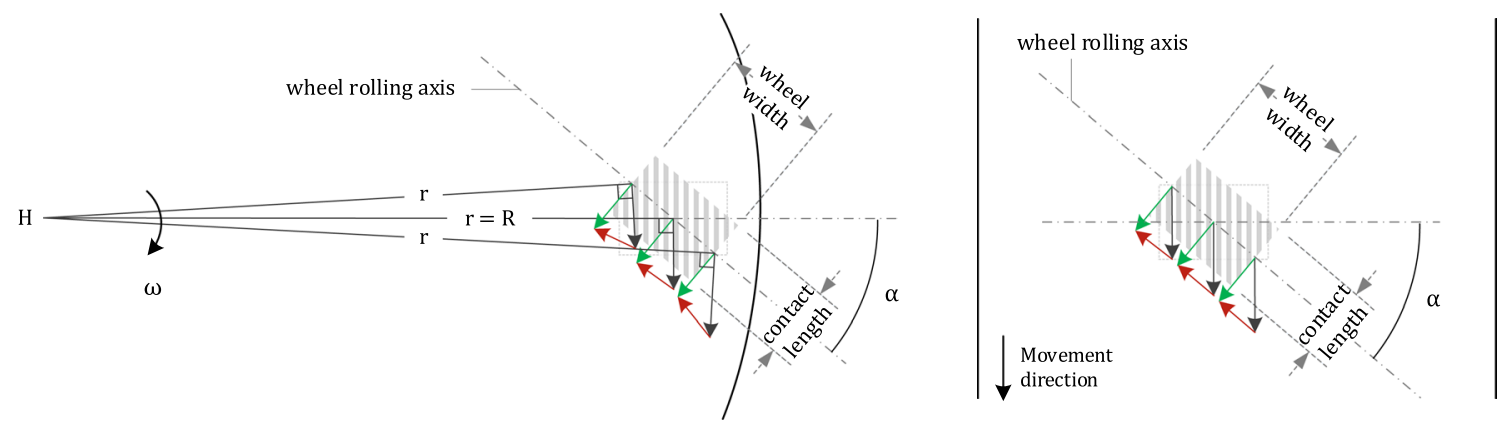

Fig. 2 Simplified schematic view of the contact area of the Grosch wheel at slip angle $\alpha\left({ }^{\circ}\right)$ with and without disk curvature. The black, green, and red vectors are disk traveling, wheel circumferential, and slip velocities, respectively; rubber deformation is disregarded (Color figure online) 
composition itself is the main factor influencing tire friction beside the tire construction and the tread pattern, see Fig. 4 [20].

To mimic the tire situation in the laboratory environment, it is required to understand what a tire experiences in the contact patch. The attempt within the previous study was to predict dry tire grip with the determination of the rubber friction in a laboratory environment. Some concepts of the interface of the tire with the road, frictional forces, slippage, and friction curves were described in two situations of longitudinal and lateral slips. Considering a simplified schematic view of tread blocks in the contact patch in longitudinal slippage in Fig. 5, tire grip is generated by the frictional forces which are created by the relative movements of these tread blocks on the ground. Slippage occurs only during cornering, braking, and accelerating when the tread block has a displacement; otherwise, the relative speed of the tire in the contact line would be zero.

Two regions can be recognized in each tire revolution: stationary and non-stationary, also known as adhesion and sliding regions according to Pacejka [23]. What every single tread block experiences in the contact area, it first starts with

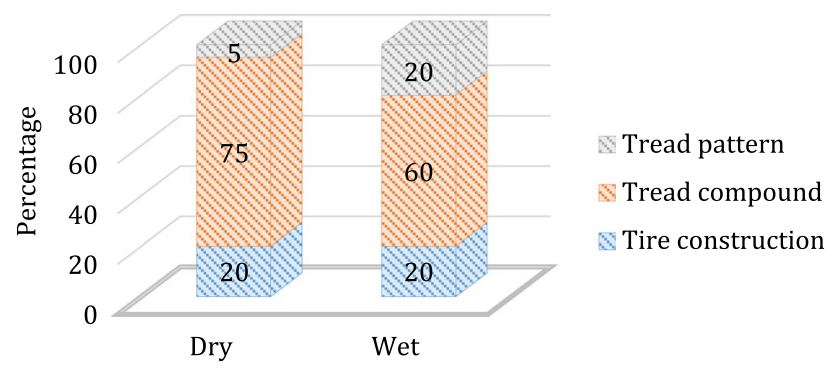

Fig. 4 The estimated contributions of the various factors on tire friction for dry/wet surfaces [20] the front non-stationary region in which the tread blocks adhere in a perpendicular direction to the counter-surface and undergo deformation and shear stress; because of the limited motion, i.e., micro-slippage in the front region of the tire, predominantly the so-called static coefficient of friction $\mu_{\mathrm{s}}$ and an adhesion friction component is involved. Then, the blocks enter into the non-stationary region and leave the contact area with a noticeable displacement under a range of frequency excitations which originate from the surface roughness of the counter-surface; it is expected to have hysteresis friction as the dominant component with the kinetic coefficient of friction $\mu_{\mathrm{k}}$ involved, which is also termed as dynamic or sliding friction. It occurs based on a relative movement of the object on the counter-surface. Without any kind of slippage and disregarding the possible rolling resistance, the tread block would leave the contact area in a perpendicular direction to the counter-surface.

The friction curve in Fig. 5 describes the transition from the stationary to the non-stationary regions by describing the friction force vs. the slip: first, the tire mostly adheres to the counter-surface; with increasing slippage, then reaches a maximum in friction force; and finally starts to slide. The ABS braking principle is commonly optimized at the highest friction. In ordinary driving, even though the tire is in motion, the major part of the contact patch relative to the ground is stationary. Therefore, both $\mu_{\mathrm{s}}$ and $\mu_{\mathrm{k}}$ are important and involved.

In the case of lateral movement, the displacement occurs in parallel to the rolling axis of the tire which will be explained in detail by exploiting a physical model: the socalled brush model. In the coming sections, the tire behaviors are qualitatively and comparatively described. Pure lateral slip is closer to the governing slip mechanism of the LAT100. A combined slip condition is not discussed in the present context.
Fig. 5 Schematic view of tire tread blocks in the contact area in longitudinal slippage with identifications of regions: stationary and non-stationary (also known as adhesion and sliding regions according to Pacejka); and friction components: adhesion and hysteresis; based on [21-23]
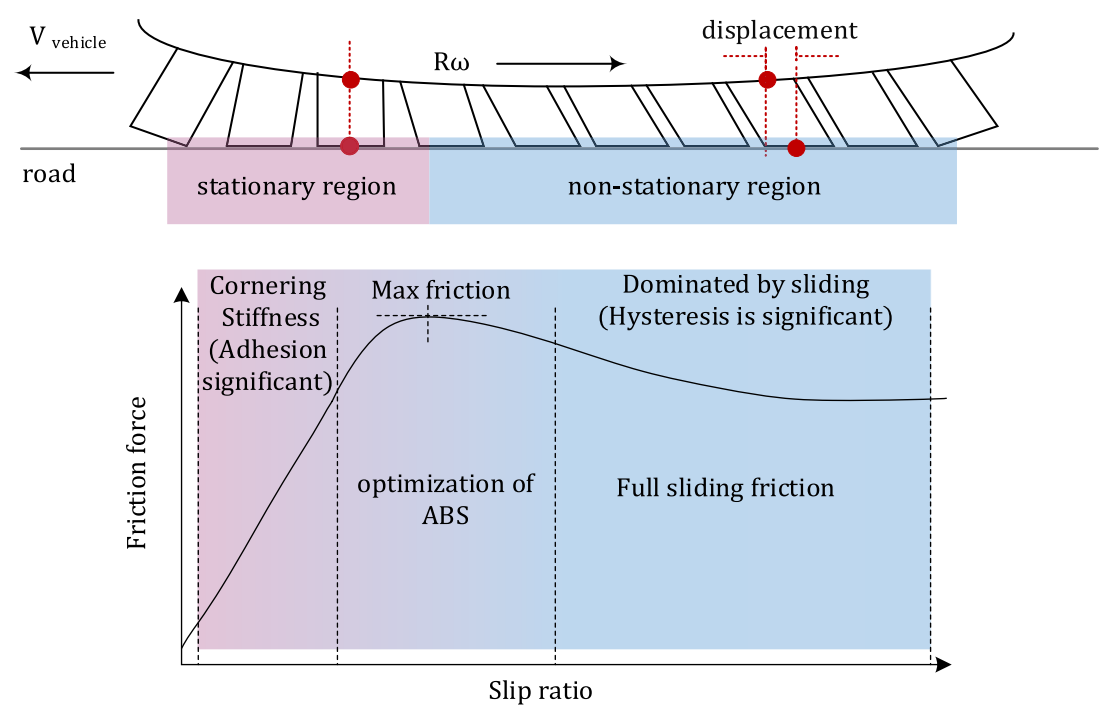


\subsection{The Tire Brush Model}

The relation between frictional force and slippage is of high importance in tire dynamics and mechanics. Figure 5 depicts schematic and graphical representations of this relationship; the mathematical relation, which is a point of great interest for researchers [4, 19, 22-32], requires sophisticated models. The concept of the 'brush' model originates from Fromm and Julien as stated by Pacejka [23]. The brush model employed in the present context describes qualitatively the main features of a slipping tire based on Pacejka and Besselink. In the present section, the nomenclature is based on Besselink [22] and the sign convention on Pacejka [23] and the equations on both. The model can be extended to better accuracy depending on the types of assumptions. Tire behavior can be described in greater detail through more complex models, computer simulations, and the finite element method [26, 33, 34].

The brush model is typically known as a row of elastic bristles attached to a rigid body called 'carcass'. Every bristle comes to the counter-surface plane, touches it, and can deflect and leave it until the next revolution. The bristles may be called tread volume or elements and their compliance corresponds to the overall elasticity of the real tire including carcass, belt, and tread layers. In the present context the brush model is described based on the following assumptions [22, 23, 30]: (i) The carcass is rigid and flattened in the contact region with a length of $2 a$; (ii) tread elements attached to carcass are elastic and the deformation of the rubber volume between the ground and the tire carcass generates the slip; (iii) the rubber part is approximated as a single row of equally-spaced deformable bristles like a brush i.e., the lateral contact width $2 b$ is assumed to be a thin slice of the tire; (iv) the bristles are compliant i.e., can deflect either in longitudinal or lateral directions parallel to the counter-surface; (v) a bristle enters the contact region perpendicular to the counter-surface; (vi) the bristle leaves the contact region after a certain time but in a presence of slip; (vii) the slip causes a bristle deflection $\varepsilon(x)(\mathrm{m})$ and force per unit length $q(x)(\mathrm{N} / \mathrm{m})$ generated in the contact point which follows Hooke's law:

$q(x)=k_{b} \cdot \varepsilon(x)$

with $k_{b}\left(\mathrm{~N} / \mathrm{m}^{2}\right)$ the bristle stiffness per unit length. This is valid either for lateral $q_{y}(x), \varepsilon_{y}(x)$ or longitudinal $q_{x}(x), \varepsilon_{x}(x)$ directions; a Cartesian coordinate system is introduced with $x$ longitudinal, $y$ lateral, $z$ perpendicular directions attached to the contact center $C$ where the coordinate system is situated; (viii) every bristle can deform independently of one another, i.e., at a specific time both stationary and non-stationary (or adhesion and sliding) regions in the contact line can occur; (ix) the longitudinal, lateral, and vertical forces are determined by summations of individual bristle contributions. The forces are the integrals over the contact length and read as follows:

$F_{x}=\int_{-a}^{a} q_{x}(x) \mathrm{d} x$,

$F_{y}=\int_{-a}^{a} q_{y}(x) \mathrm{d} x$,

$F_{z}=F_{N}=\int_{-a}^{a} q_{z}(x) \mathrm{d} x$.

Figure 6 depicts the tire brush model according to the mentioned assumptions. Pure slip conditions can occur:

1. When the velocity of travel, vector $V$ shows an angle $\alpha$ with the symmetry plane of the tire normal to the rolling axis; it creates lateral slip;

2. When the forward component of the wheel velocity $V_{x}=V \cos \alpha$ is not equivalent to $V_{\mathrm{r}}=r_{\mathrm{e}} \Omega$, where $\Omega$ $(\mathrm{rad} / \mathrm{s})$ is the angular speed of the tire; it creates longitudinal slip.

The tip of each bristle moves from the leading to the trailing edge and stays in contact with the counter-surface as long as friction allows in each revolution. The deflections of the bristles increase in a direction parallel to the velocity $V$ towards the trailing edge. This velocity vector could be parallel to $V_{x}$ in

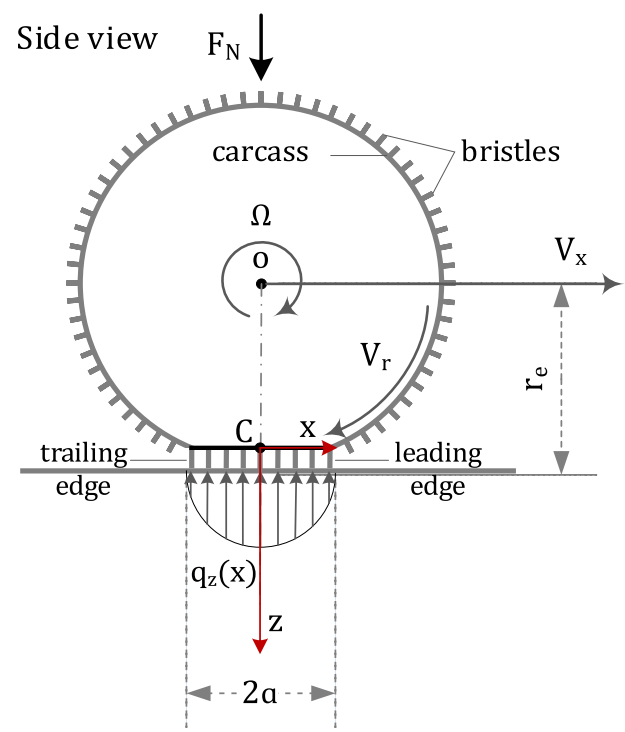

Fig. 6 The tire brush model drew according to the assumptions and [22] 
longitudinal slip or form an angle with $V$ in the case of lateral slip. The base points of the bristles fixed to the carcass move backward with a velocity of $V_{\mathrm{r}}$ relative to the contact center $C$; while with respect to the counter-surface, the base points travel with a slip speed $V_{\mathrm{s}}$, see Fig. 7 .

By assuming a parabolic function for $q_{z}(x), F_{z}$ in Eq. (7) can be obtained by inserting the following expression:

$q_{z}(x)=\frac{3 F_{z}}{4 a}\left(1-\left(\frac{x}{a}\right)^{2}\right)$

The maximum deflection of the bristles $\varepsilon_{\max }(x)$ is limited by the presence of the coefficient of friction $\mu$, assumed constant. The constant friction coefficient in the current model might seems primitive; however, it is good enough to demonstrate the basic differences between the tire test modalities in comparison with the Grosch wheel. The magnitude of the generated frictional force per unit length $q(x)$ is defined according to Coulomb's equation:

$|q(x)| \leq \mu q_{z}(x)$.

Pure longitudinal slip-Side view

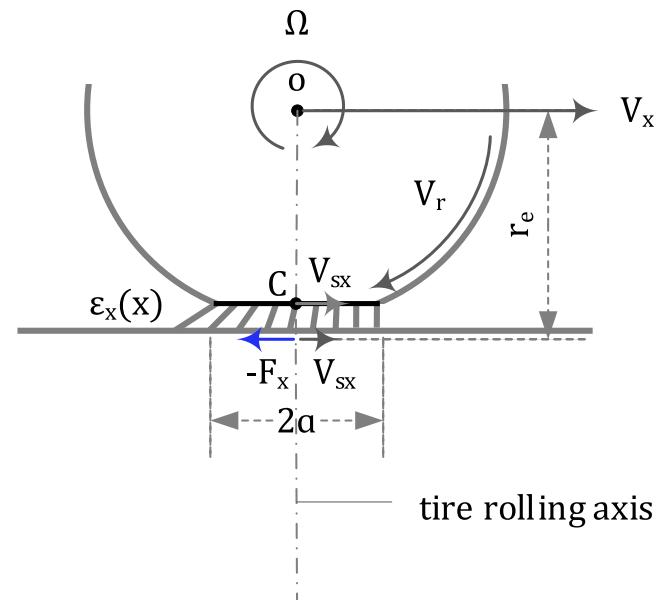

Pure lateral slip-Top view

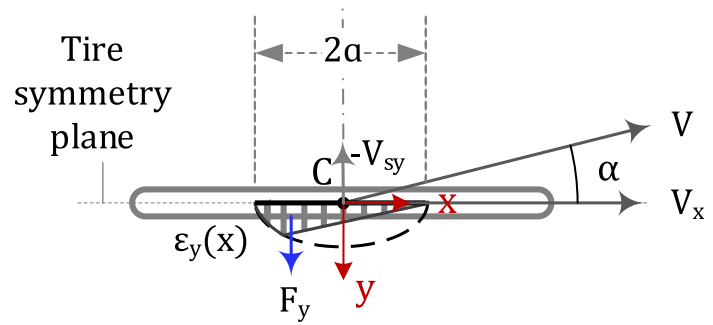

Equation (9) is valid whether the bristle moves in $x$ or $y$ direction, i.e., longitudinal $q_{x}(x)$ or lateral $q_{y}(x)$ direction. From Eqs. (4) Hooke's law and (9) Coulomb's law, the maximum bristle deflection $\varepsilon_{\max }(x)$ can be derived in Eq. (10) and has a parabolic shape because it is a function of $q_{z}(x)$ :

$\left|\varepsilon_{\max }(x)\right|=\frac{\mu q_{z}(x)}{k_{b}}$

This concept is a good basis for the following discussions of the mathematical calculations of the frictional forces and 'break-away' point which is the transition between the stationary and non-stationary regions. In the next section, they will be discussed for two scenarios of pure lateral and longitudinal slip conditions.

\subsubsection{Pure Lateral (Side) Slip}

In the stationary region, the contact line between the bristles and the counter-surface is straight, the bristles follow the straight line of contact which is parallel to $V$, the velocity of travel. By applying the slip angle $\alpha$, at the moment that the straight line of contact intersects with the maximum deflection parabola $\varepsilon_{\max }$, the non-stationary region begins from the intersection or the break-away point in Fig. 8A. The bristles coincide with the parabola for the maximum possible deflection. The deflection is not linear anymore and is curved indicating that available frictional forces become lower than the forces that would have continued along the straight line, Fig. 8B. Due to asymmetry of the deflection, a self-aligning torque or moment $M_{z}$ arises. The moment is the product of multiplying the lateral force $F_{y}$ by the moment arm, or the 'pneumatic trail' $t_{\mathrm{p}}$, which is the distance of the lateral force line of action from point $C$. As the slip angle increases, the deformation profile becomes more symmetric. The point of intersection shifts to the leading edge, the lateral force escalates, and $t_{\mathrm{p}}$ decreases; until the contact line becomes the tangent to the parabola at the foremost of the leading edge, Fig. 8C. The deformation becomes fully symmetric, and the stationary (adhesion) region disappears and full sliding occurs. The friction curve turns flat, the $t_{\mathrm{p}}$ becomes zero, and the self-aligning moment vanishes. By further increasing the slip angle, the situation for bristles has to stay theoretically unchanged, see Fig. 8D.

The mathematical equations for the above description of the pure lateral slip are derived for a steady-state situation $\partial \varepsilon / \partial s=0$, i.e., deflection does not change with distance $s$. Consider $\Delta t$ the time that the bristle already spent in the contact region:

$\Delta t=\frac{a-x}{V_{\mathrm{r}}}$

Fig. 7 Pure longitudinal and lateral slip conditions, based on [22, 23] 
A

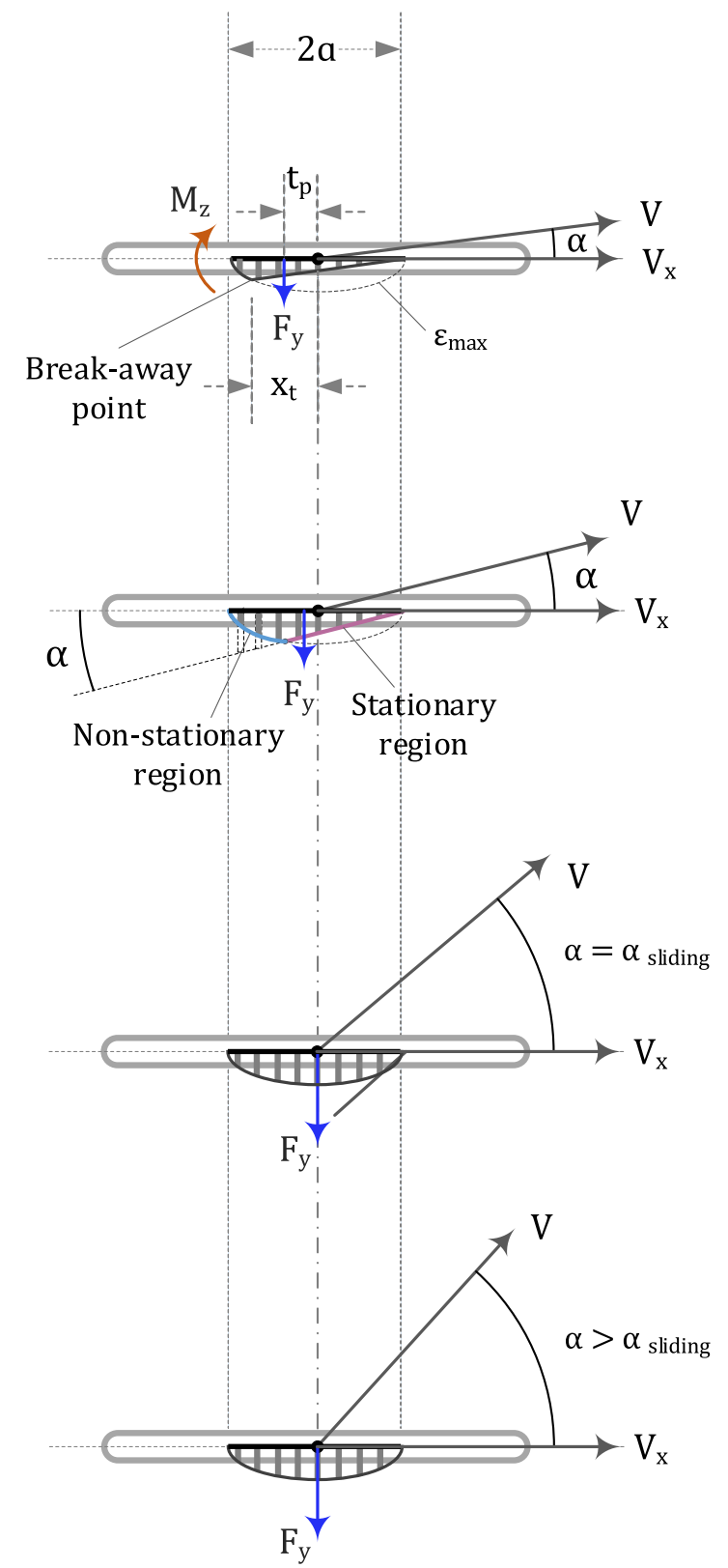

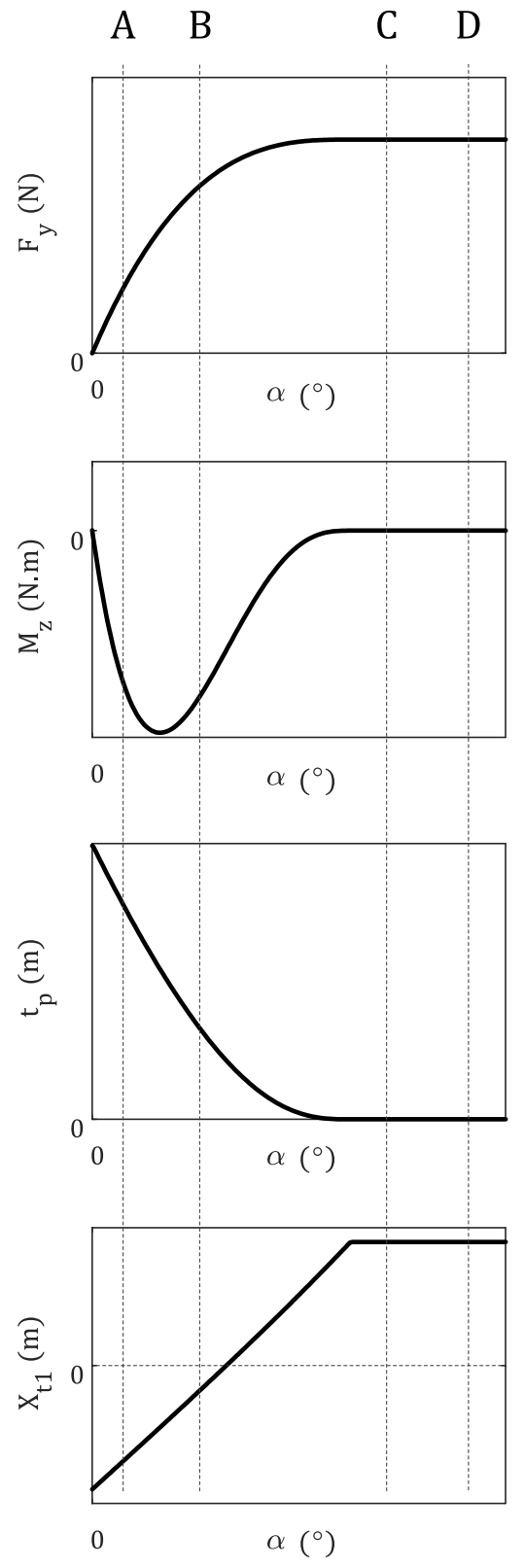

Fig. 8 Pure lateral slip condition and resulting force and aligning moment by increasing slip angle $\alpha$, based on [22, 23]

then the pure lateral deflection derived from the trigonometry of velocities in the contact region [22] is as follows, see also Fig. 7:

$\varepsilon_{y}(x)=-V_{\mathrm{sy}} \Delta t=(a-x) \tan \alpha$.

Based on the last assumption of the model in the previous section, the lateral or cornering force $F_{y}$ is the integral of $q_{y}(x)$ over the contact length $2 a$; by substituting Eqs. (4) and (12) for very small slip angle, it results:
$F_{y}=\int_{-a}^{a} q_{y}(x) \mathrm{d} x=k_{b} \int_{-a}^{a} \varepsilon_{y}(x) \mathrm{d} x=k_{b} \tan \alpha \int_{-a}^{a}(a-x) \mathrm{d} x$.

By solving the integral for very small $\alpha \approx \tan \alpha$, the relationship between frictional force and slippage, i.e., $F_{y}$ as a function of $\alpha$ in the stationary region follows the expression below: 
$F_{y}=2 k_{b} a^{2} \alpha=C_{\mathrm{F} \alpha} \alpha$,

where $C_{F \alpha}(\mathrm{N} / \mathrm{rad})$ is the cornering stiffness. Note that the cornering stiffness is the slope of $F_{y}$ vs. $\alpha$ :

$C_{F \alpha}=\left(\partial F_{y} / \partial \alpha\right)_{\alpha \rightarrow 0}$

which depends on tread stiffness and the length of contact $2 a$. Subsequently, the self-aligning moment $M_{z}$ is calculated with the moment arm:

$M_{z}=\int_{-a}^{a} q_{y}(x) x \mathrm{~d} x=-\frac{2}{3} k_{b} a^{3} \alpha=C_{\mathrm{M} \alpha} \alpha$,

where $C_{\mathrm{M} \alpha}(\mathrm{Nm} / \mathrm{rad})$ is the self-aligning stiffness. Accordingly, the pneumatic trail $t_{\mathrm{p}}$ can be calculated using Eqs. (14) and (16) as follows:

$M_{z}=F_{y} \cdot t_{p} \rightarrow\left(t_{p}\right)_{\alpha \rightarrow 0}=\frac{1}{3} a$.

These calculations were made for the stationary region. To find the distance of the break-away point $x_{t}$ to the center $C$ (Fig. 8), the linear bristle deflection in the stationary region has to be equal to the maximum of the parabolic bristle deflection. Hence, from Eqs. (10) and (12), two solutions are obtained for $x_{t}$ :

$\left.\varepsilon_{y}(x)\right|_{\text {at } x_{t}}=\left.\varepsilon_{y \max }(x)\right|_{\text {at } x_{t}} \Rightarrow$

solution $1: x_{t 1}=\frac{4 k_{\mathrm{b}} a^{3}}{3 \mu F_{z}}(\tan \alpha)-a$,

solution $2: x_{t 2}=a$

Solution $x_{t 1}$ is the break-away point, describing that from $a$ to $x_{t 1}$ is the stationary region and from $x_{t 1}$ to $-a$ is the nonstationary one. If $x_{t 1}=a$, the sliding slip angle $\alpha_{\text {sliding }}$ can be deduced:

$\alpha_{\text {sliding }}=\tan ^{-1}\left(\frac{3 \mu F_{z}}{2 k_{\mathrm{b}} a^{2}}\right)$

This is the slip angle when the stationary region vanishes and full sliding occurs, where solution 2 is indicated, Fig. 8C. Similar to Eq. (13), $F_{y}$ and $M_{z}$ can be written for the contact length when both regions exist. For the stationary region Eq. (4) and the non-stationary region Eq. (9) which are relevant, hence $F_{y}$ is the summation of two integrals:

$F_{y}=\frac{3 \mu F_{z}}{4 a} \int_{-a}^{x_{t}}\left(1-\left(\frac{x}{a}\right)^{2}\right) \mathrm{d} x+k_{b} \int_{x_{t}}^{a}(a-x) \tan \alpha \mathrm{d} x$.

Correspondingly, by considering the torque arm, the aligning moment becomes
$M_{z}=\frac{3 \mu F_{z}}{4 a} \int_{-a}^{x_{t}}\left(1-\left(\frac{x}{a}\right)^{2}\right) x d x+k_{b} \int_{x_{t}}^{a}(a-x) \tan \alpha x d x$.

The solutions of the integrals of the above expressions depend on $x_{t}$.

- If $x_{t}=x_{t 2}=a$, it indicates that the $\alpha \geq \alpha_{\text {sliding }}$, then

$F_{y}=\mu F_{z}$

$M_{z}=0$

- If $x_{t}=x_{t 1}$, i.e., $\alpha \leq \alpha_{\text {sliding, }}$, then the integral has to be solved based on $x_{t 1}$ in Eq. (18), which results in

$$
\begin{aligned}
& F_{y}=\mu F_{z} \tan \alpha\left(3 \theta-3 \theta^{2} \tan ^{2} \alpha+\theta^{3} \tan ^{3} \alpha\right), \\
& M_{y}=-\mu F_{z} a \theta \tan \alpha\left(1-3|\theta \tan \alpha|+3(\theta \tan \alpha)^{2}-(\theta \tan \alpha)^{3}\right),
\end{aligned}
$$

where the parameter $\theta$ is defined as follows [23]:

$\theta=\frac{2 k_{b} a^{2}}{3 \mu F_{z}}$.

And subsequently, $t_{\mathrm{p}}$ holds as follows:

$t_{p}=\frac{a}{3}\left(\frac{1-3|\theta \tan \alpha|+3(\theta \tan \alpha)^{2}-(\theta \tan \alpha)^{3}}{\left(1-|\theta \tan \alpha|+\frac{1}{3}(\theta \tan \alpha)^{2}\right)}\right)$

\subsubsection{Pure Longitudinal Slip}

The basic principles for lateral and longitudinal slip are similar, except that the longitudinal deflection follows Eq. (28) [22]:

$\varepsilon_{x}(x)=-V_{\mathrm{s} x} \Delta t=(a-x) \frac{\kappa}{1+\kappa}$,

where $\kappa$ is the slip ratio as described in the previous paper. According to the coordinate system, it holds that

$\kappa=-\frac{V_{s x}}{\left|V_{x}\right|}=-\frac{V_{x}-r_{e} \Omega}{\left|V_{x}\right|}$.

From the similarity between the bristle deflections in $x$ and $y$ directions, i.e., Equations (12) and (28), it can be deduced that by replacing $\tan \alpha$ in Eq. (24) for $F_{y}$ by $\kappa /(1+\kappa)$, the force $F_{x}$ can be derived as follows: 
$F_{x}=3 \mu F_{z} \theta \frac{\kappa}{1+\kappa}\left(1-\left|\theta \frac{\kappa}{1+\kappa}\right|+\frac{1}{3}\left(\theta \frac{\kappa}{1+\kappa}\right)^{2}\right)$.

And accordingly the break-away point $x_{t 1}$ can be described as follows:

$x_{t 1}=\frac{4 k_{b} a^{3}}{3 \mu F_{z}}\left|\frac{\kappa}{1+\kappa}\right|-a$.

In Sect. 4, the implementation, possible improvement, and limitations of the model for the LAT100 compared with the tire are investigated.

\subsubsection{Accuracy of the Brush Model}

The presented model is already complex but still contains numerous assumptions. Besselink [22] calculated the errors of the presented brush model compared to actual tire measurements for $F_{y}, F_{x}$, and $M_{z}$ in pure slip conditions to be $15 \%, 11 \%$, and $73 \%$, respectively. The error for the aligning moment $M_{z}$ is notably high. To reduce the errors and improve the accuracy of the presented model for a real tire, the assumptions have to be refined, see later in Sect. 4.3.

\section{Implementation of the Brush Model for the Grosch Wheel}

\subsection{Model Inputs}

The presented brush model is now used to explain the physics and dynamics of the Grosch wheel as the test specimen of the LAT100 machine as shown in Fig. 9. The Grosch sample specification is illustrated in Fig. 1; a solid rubber wheel with no air inside, while an actual tire is pneumatic with specific construction. The internal structure of a real tire is much more complicated compared to the simple Grosch rubber wheel. For the Grosch wheel, the parabolic pressure distribution is considered as a basis to start with the model implementation (Table 1).

Due to the geometry of the wheel-shaped sample, the contact length $2 a$ depends on the vertical wheel deflection $\delta$ as shown in Fig. 3. By rising the normal load, $\delta$ increases. Schallamach and Turner [31] suggested a proportionality between the square of the contact length of the wheel with the counter-surface, and the ratio of normal load divided by Young's modulus of the rubber compound, i.e., $a^{2} \propto\left(F_{\mathrm{N}} / E\right)$. Besselink [22] introduced a pragmatic approach that matches with the measurement data of vertical
Fig. 9 The velocities and the resultant friction forces of the Grosch wheel sample on the LAT100 counter-surface disk at slip angle $\alpha$ according to brush model and sign convention of Pacejka

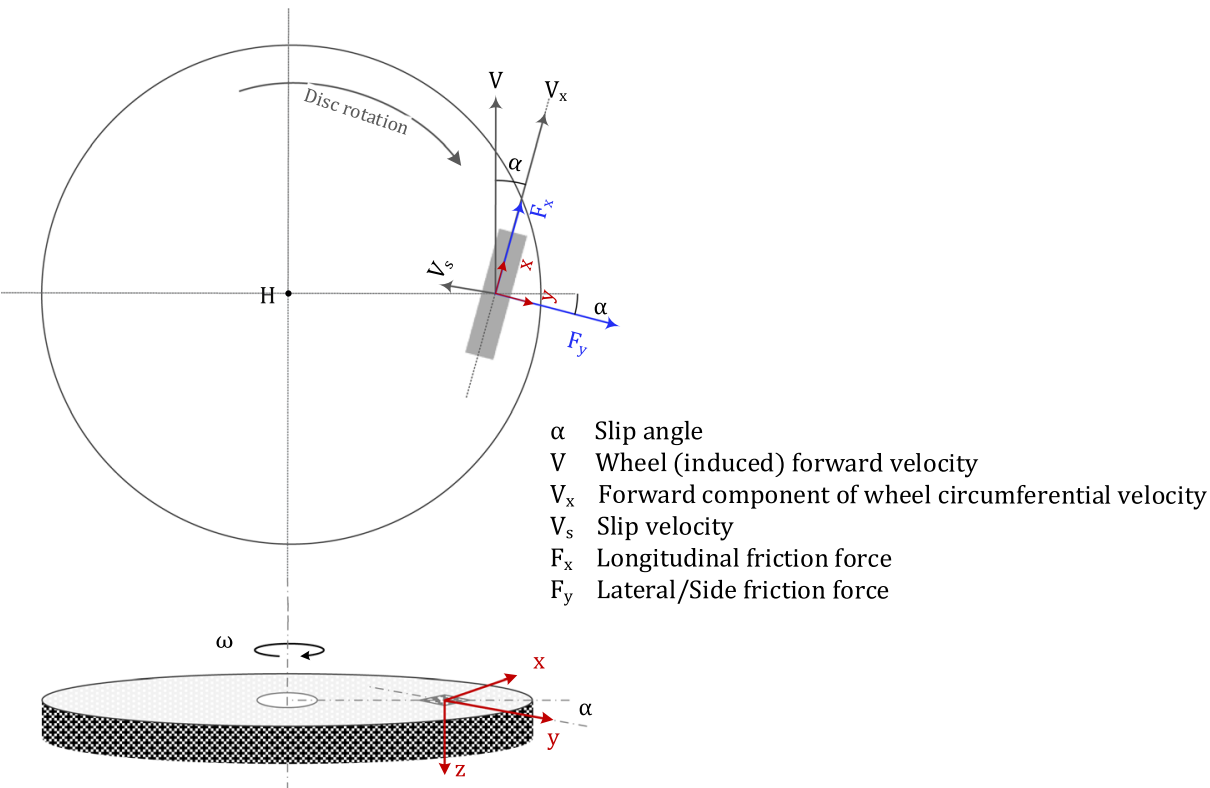

Table 1 Alteration of contact length with normal load, measured with pressure-sensitive film, type LLLW; 2a is identified

\begin{tabular}{|c|c|c|c|c|c|}
\hline Load & $35 \mathrm{~N}$ & $55 \mathrm{~N}$ & $75 \mathrm{~N}$ & $95 \mathrm{~N}$ & $115 \mathrm{~N}$ \\
\hline Compound A & 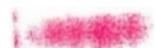 & & & & \\
\hline
\end{tabular}


deflections of tires. In the present study, the alteration of the contact length was determined with the normal load using Fuji film pressure-sensitive film in a so-called 'static' measurement in which a 'momentary' pressure on the nonmoving wheel is applied in a way that the normal load was raised gradually for five seconds and then maintained at this level for a further five seconds. Figure 10 is a graphical representation of the variations of the experimental values of the contact length $2 a$ and width $2 b$ vs. normal load for the Grosch wheel with a typical tread compound A (the properties of compound A are listed in Table 4 in "Appendix"). The variation of the width $2 b$ with load is neglected, see Table 1 and Fig. 10.

The values for bristle stiffness $k_{b}$ are taken as the storage moduli $G$ of the rubber compounds from frequency sweep measurements with Dynamic Mechanical Thermal Analyses (DMTA) at $0.1 \%$ strain, assuming the geometry factor of the bristles is of order unity. The coefficient of friction $\mu$ is taken from the maximum value of the friction curve obtained with the LAT100. A summary of the modeling parameters for two compounds $\mathrm{A}$ and $\mathrm{E}$ is listed in Table 2.

\subsection{Model Outputs}

Modeling was performed in two parts:

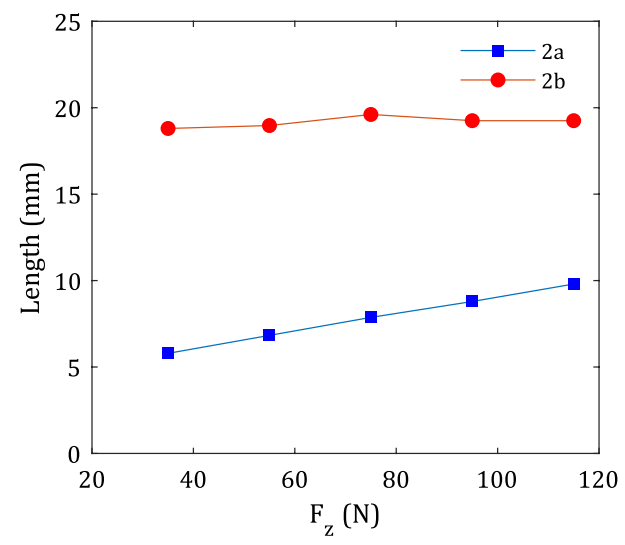

Fig. 10 Alteration of the experimental values of the contact length $2 a$ and width $2 b$ with varying normal load for the Grosch wheel with tread compound A
First, half of the contact length $a$ was taken from 'static' measurements of contact area as illustrated in Fig. 10; the input values for compound A are presented in Table 2. Figure 11 represents a comparison of the modeling data and the measurements with the LAT100: the lateral force $F_{y}$ vs. slip angle $\alpha$, Eq. (24). Although the stiffness of the rubber compound and normal load were considered in the evaluation of the 'static' $a$, the outcome of the model is far from the experimental data: the dashed vs. the solid red curves. The model is very sensitive to bristle stiffness, and therefore, the rubber modulus governs the friction curve up to the $\alpha_{\text {sliding }}$. The model accuracy can be altered significantly by the $G$ value: for instance, assuming an hypothetical value of $15 \mathrm{MPa}$ for $G$, (Fig. 11 with the dotted curve). Hence, obtaining a proper value for $G$ is very important, as the modulus can change based on the type of test and its condition, see Table 4 in "Appendix".

Second, an estimate of the $a$ value is obtained using the LAT100 experimental data which is expected to represent the 'dynamic' contact length. From Eq. (14) at a very small $\alpha<1^{\circ}$, half of the contact length $a$ can be extracted:

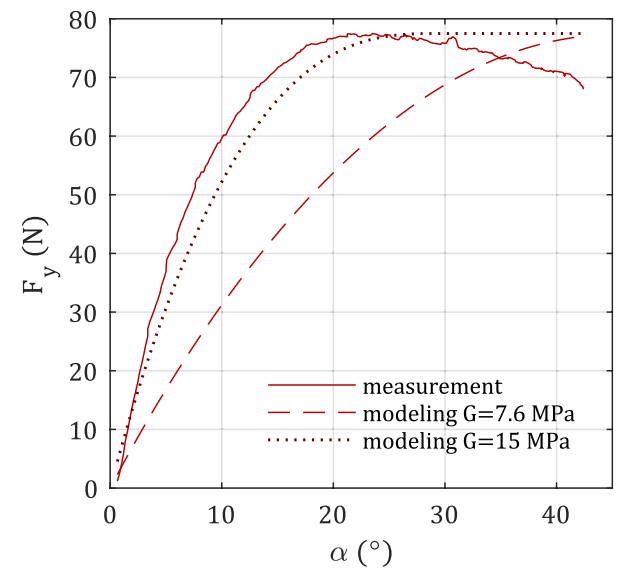

Fig. 11 Measurement results and modeling outputs of $F_{y}$ vs. $\alpha$ for $a$ 'static'; the modeling inputs are taken from Table 2. The dotted curve shows the influence of the bristle stiffness or $G$ value of the rubber compound on modeling for the Grosch wheel with tread compound A
Table 2 Modeling parameters for the Grosch wheel from compound $\mathrm{A}$ and $\mathrm{E}$, at speed $3.7 \mathrm{~km} / \mathrm{h}$

\begin{tabular}{lllll}
\hline Parameter & Symbol & Compound A & Compound E & Unit \\
\hline Wheel radius & $r_{\mathrm{w}}$ & 41 & 41 & $\mathrm{~mm}$ \\
Wheel width & $b$ & 9 & 9 & $\mathrm{~mm}$ \\
Bristle stiffness & $k_{b}$ & 7.6 & 4.5 & $\mathrm{~N} / \mathrm{mm}^{2}$ or MPa \\
$\begin{array}{l}\text { Coefficient of friction } \\
\text { Half of the contact length at }\end{array}$ & $\mu$ & 1.2 & 1 & - \\
$\quad$ load 65 N & $a$ & 3.7 & 3.1 & $\mathrm{~mm}$ \\
\hline
\end{tabular}




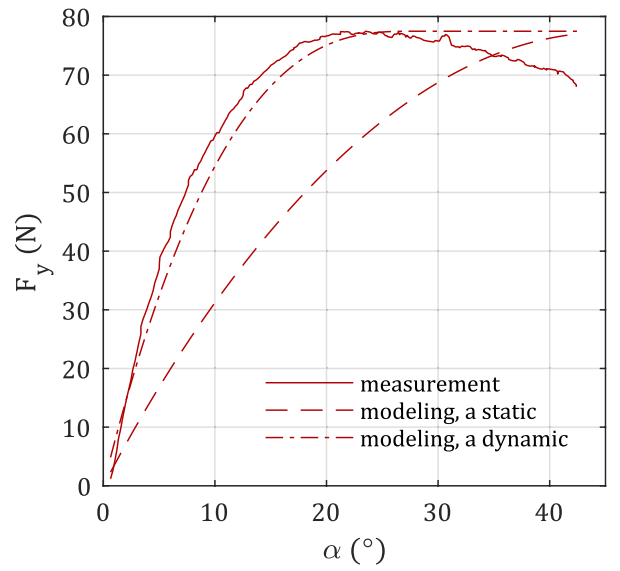

Fig. 12 Lateral force $F_{y}$ vs. slip angle $\alpha$ for compound A; implementation of the ' $a$ dynamic' compared to the ' $a$ static' with LAT100 measurement data at $G$ of $7.6 \mathrm{MPa}$

$a=\sqrt{\frac{C_{\mathrm{F} \alpha}}{2 k_{b}}}$

By implementing the 'dynamic' approximate of $a$ in the model, the outcome is presented in Fig. 12 in comparison with the 'static'; the modification of $a$ improves the model accuracy for the prediction of $F_{y}$ remarkably. Note that beyond $\alpha_{\text {sliding, }}$, the model follows Eq. (22) where the Coulomb approximation of the sliding region is clearly poor.

Figure 13 illustrates the outcome of the model, lateral force $F_{y}$ vs. slip angle $\alpha$, for a softer compound $\mathrm{E}$ than compound A, also presented in Table 4 in "Appendix"; the modeling parameters for compound $\mathrm{E}$ are listed in Table 2. There is a vertical shift upwards for the experimental friction curve $F_{y}$ vs. $\alpha$ at $\alpha=0^{\circ}$ for compound E, which could indicate the presence of a pseudo-camber angle. The wheel camber or inclination angle $\gamma$ is the angle between the planes of the wheel and the normal to the counter-surface. The shift can stem from non-uniformity of the vulcanized sample. The Grosch wheel sample is $19 \mathrm{~mm}$ thick which inevitably leads to a gradient in crosslink density and consequent inhomogeneity of the specimen [11]. Still, by using the $a$ 'dynamic' in the calculation, the deviation of the modeling output for compound $\mathrm{E}$ from the measurement result is larger than for compound A. Figure 14 shows a comparison of the lateral forces $F_{y}$, aligning moments $M_{z}$, and pneumatic trails $t_{\mathrm{p}}$ vs. slip angle $\alpha$ for compounds $\mathrm{A}$ and $\mathrm{E}$.

However, there is a subtle point concerning introducing the $a$ 'dynamic' in Eq. (32). The model outcome $F_{y}$ vs. $\alpha$ becomes insensitive to variation of the $G$ value for a particular compound but not the $M_{z}$ and $t_{\mathrm{p}}$. The model outcomes are presented for various hypothetical $\mathrm{G}$ values for compound $\mathrm{A}$ in Fig. 15. The G value of 7.6 MPa was taken from Table 4;

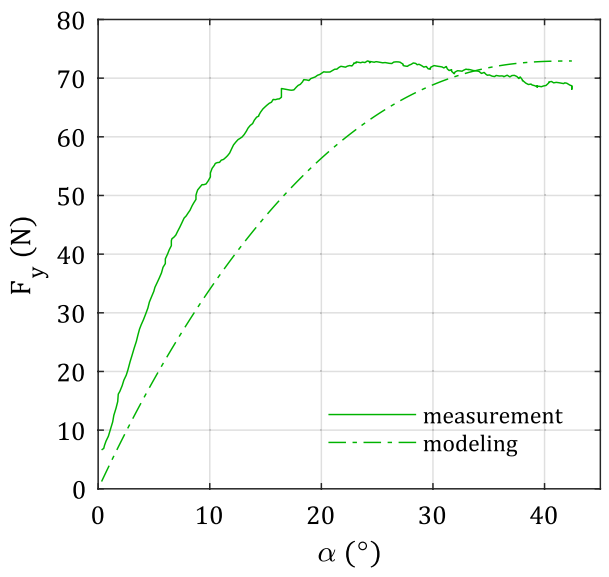

Fig. 13 lateral force $F_{y}$ vs. slip angle $\alpha$ for Grosch wheel with compound $\mathrm{E}, a$ 'dynamic' is used

the data are presented as well for $3.8 \mathrm{MPa}(0.5 \mathrm{G})$ and 15.2 (2G). No change of $F_{y}$ can be observed. This can be traced back to the $\alpha_{\text {sliding }}$ value calculated by Eq. (19). By substituting Eq. (32) in (19), the $\alpha_{\text {sliding }}$ value becomes invariant to the $\mathrm{G}$ modulus, it reads as follows:

$\alpha_{\text {sliding }}=\tan ^{-1}\left(\frac{3 \mu F_{z}}{C_{\mathrm{F} \alpha}}\right)$.

While the self-aligning moment $M_{z}$ and the pneumatic trail $t_{\mathrm{p}}$ differ based on the $G$ values, the stiffer compound has a smaller pneumatic trail and needs a lower aligning torque. Based on this outcome, it may be concluded that $F_{y}$ before reaching the $\alpha_{\text {sliding }}$ point is not a function of the tread stiffness. However, in reality, the tread stiffness affects the friction curve and $\alpha_{\text {sliding }}$ value as elaborated in the previous study [1]. This is a limitation of the current assumptions for the model. To get closer to the experimental data, some ways are explained in the next section.

\subsection{Potential Model Improvements}

As explained in Sect. 3, the assumptions for the model may be refined to reduce the errors and improve the accuracy, e.g., by introducing

i. an elastic carcass;

ii. non-linear bristle deflections;

iii. the rubber volume as multiple rows of deformable bristles;

iv. realistic pressure distribution in the contact area;

v. the dependency of the coefficient of friction $\mu$ on slip speed and normal load by implementing available friction theories; 

ing moment $M_{z}$, and pneumatic trail $t_{\mathrm{p}}$ vs. slip angle $\alpha$ for com-
Fig. 14 Lateral force $F_{y}$, alignpounds $\mathrm{A}$ and $\mathrm{E}$
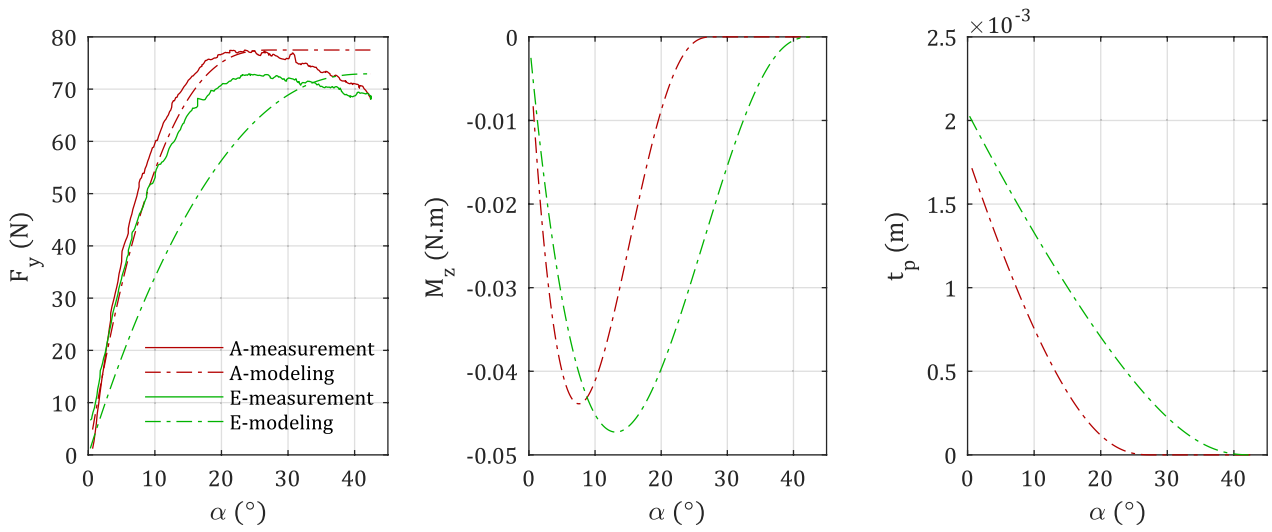

Fig. 15 Lateral force $F_{y}$, aligning moment $M_{z}$, and pneumatic trail $t_{\mathrm{p}}$ vs. slip angle $\alpha$ for compound $\mathrm{A}$; at various hypothetical bristle stiffness of $0.5 \mathrm{G}, \mathrm{G}$, and $2 \mathrm{G}$ presented with colors dark to light green, respectively; $G$ value is $7.6 \mathrm{MPa}$
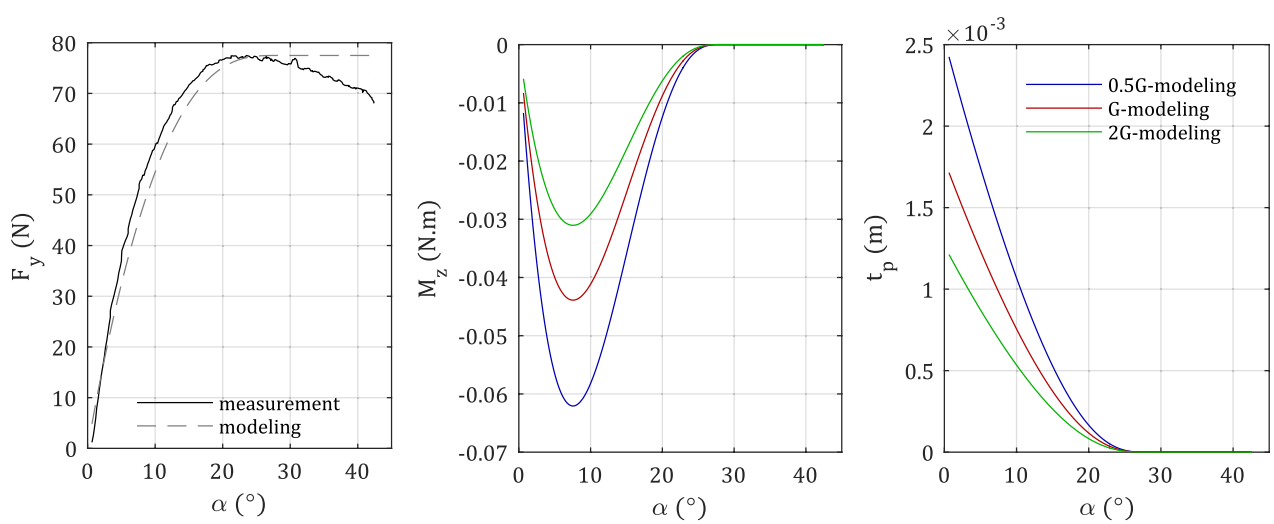

vi. deal with non-uniform carcass deflection;

vii. inclusion of inclination (camber) angle or path curvature.

In 'Treadsim' which is an improved brush model by Pacejka [35], some of the above improvements have been further developed for tires. The modeling in the present study was performed to create a basic understanding of the physics and dynamics of the Grosch wheel in the contact patch and attribute them to friction components and mechanisms. The authors are of the opinion that even a basic model with constant $\mu$ should display the reason behind for no correlation between tire $\alpha$ and $\kappa$ sweep tests of the previous study which are discussed in the next section.

\subsection{Attribution of Friction Components}

With the presented model, some insights into the dynamics and physics of the Grosch wheel as the specimen for the LAT100, the newly developed tribometer, were obtained. The attempt was not purely to discover the most accurate model, but to acquire a deeper understanding of the slippage regions, i.e., stationary and non-stationary. This bridges the gap between wheel dynamics and rubber friction mechanisms. It creates a clear and intelligible profundity of understanding friction curves in connection with rubber characterization and frictional mechanisms.

The stationary and non-stationary regions were identified in the model, as marked in Figs. 5 and $8 \mathrm{~b}$ with magenta and blue colors, respectively. Each region encompasses specific contributions of frictional mechanisms, where different components are expected to govern and play the main role. In the stationary region, the tread deforms and stresses under shear which predominantly contributes to the adhesive friction component. The classical description of long-chain rubber polymers at the interface with a counter-surface dictates that by applying shear stress on the tread block, the rubber chains stretch, detach, relax, and re-attach to the countersurface whereupon the cycle repeats itself [36]. In the nonstationary region due to the displacement, the tread blocks are subjected to a range of frequency excitations originating from the asperities of the counter-surface, where viscoelastic or hysteresis friction could prevail. This can be measured with a sliding friction measurement and also Persson's friction theory can calculate the viscoelastic part. The adhesion contribution can be obtained with a separate measurement designed for adhesion evaluation [37].

The friction curve can be split into three main sections concerning characteristic shape factors: linear, transitional, and frictional, see Fig. 16. The linear section indicating 
cornering stiffness represents basic linear handling and stability of the tire; the transitional section is mainly affected by tread stiffness; the frictional range depends primarily on the viscoelastic properties. The advantage of this categorization of the friction curve is that it provides information regarding the slippage regions and accordingly distinguishes the prevailing friction components.

Full sliding starts at the peak of the friction curve, and after that, the Coulomb equation in the model is a poor representation. For comparison of $\alpha$ and $\kappa$ sweep tests, the main focus has to be dedicated to the sections before the peak occurs where the contributions of both regions, i.e., stationary and non-stationary, envisage a high likeliness for different friction mechanisms. It is noteworthy that in reality and in ordinary driving, the passenger car tire gets an angle of $2^{\circ}-6^{\circ}$ during cornering in bends while the peak typically occurs at $4^{\circ}-7^{\circ}[21]$. Therefore, both regions need to be considered for tire friction. From this, it is clear why wider tires provide more grip because of the possibility to enlarge the stationary region which results in enhanced static friction.

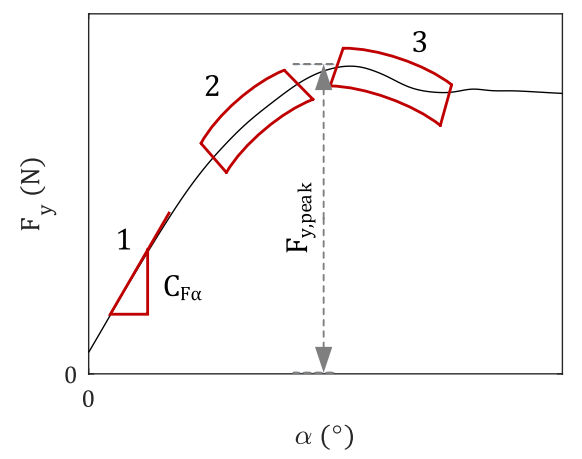

Fig. 16 Characteristic shape factors of the friction curve: 1. linear, 2. transitional, and 3. frictional; after Pacejka [38]

\subsection{Comparison of Tire $a$ and $\kappa$ Sweep Tests}

The tests modalities need to be compared at an equivalent scale or measure. This measure is defined as 'theoretical slip' for longitudinal and lateral slip conditions. Table 3 shows a comparison between both slip types.

Theoretically, by assuming an equal coefficient of friction and bristle stiffness at both pure slip types, i.e., supposedly $\mu_{x}=\mu_{y}$ and $k_{b x}=k_{b y}$, then

- The equation for the longitudinal stiffness $C_{\mathrm{F} \kappa}$ is identical to the cornering stiffness $C_{\mathrm{F} \alpha}$;

- The theoretical slip at sliding occurs at the same $\theta$ for both types.

Hence, the equality of the friction curves is described in Fig. 17.

In reality, a notable discrepancy between the measured values for longitudinal and cornering stiffness is observed. Pacejka [23] indicated that $C_{\mathrm{F} K}$ is typically $50 \%$ larger than $C_{\mathrm{F} \alpha}$ due to the lateral (torsional) compliance of the carcass of an actual tire. Also cornering stiffness may differ with tire vertical load, from 6 to 30 times according to Pacejka. Besselink [22] plotted the measured values of $C_{\mathrm{F}}$ in longitudinal and lateral slip conditions vs. vertical load. The $C_{\mathrm{F} K}$ values were larger than $C_{\mathrm{F} \alpha}$ around by one order of magnitude.

Still, it is expected that the qualitative similarity of both pure slip characteristics remains. By comparing actual tire data of $\alpha$ and $\kappa$ sweep tests from the previous study [1], the portions of stationary and non-stationary regions in each test can be approximated by the model. An example of the comparison between both $\alpha$ and $\mathrm{\kappa}$ tests for the same tread compound is shown in Fig. 18 where all other variables remained the same. The results are plotted vs. the characteristic slip parameters and the theoretical slips for the modeling and the measurement data. The right-side plots of each row describe the disparity between the $\alpha$ and $\kappa$ tests: at equivalent theoretical slip values, the curves show different characteristic shapes (see Fig. 16). This becomes more pronounced by
Table 3 Comparison of the equations derived from the brush model in longitudinal and lateral slip conditions

\begin{tabular}{lll}
\hline Slip type & Pure longitudinal $(x$ direction) & Pure lateral $(y$ direction) \\
\hline Characteristic parameter & $\kappa$ & $\alpha$ \\
Slip definition & $\kappa=-\frac{V_{s x}}{\left|V_{x}\right|}$ & $\tan \alpha=-\frac{V_{s y}}{\left|V_{x}\right|}$ \\
Bristle deflection & $\varepsilon_{x}(x)=-V_{s x} \Delta t=(a-x) \frac{\kappa}{1+\kappa}$ & $\varepsilon_{y}(x)=-V_{s y} \Delta t=(a-x) \tan \alpha$ \\
Theoretical slip, $s$ & $s_{x}=\frac{\kappa}{1+\kappa}$ & $s_{y}=\tan \alpha$ \\
At small slip & $F_{x}=C_{\mathrm{F} \kappa} \kappa, C_{\mathrm{F} \kappa}=2 k_{b} a^{2}$ & $F_{y}=C_{\mathrm{F} \alpha} \alpha, C_{\mathrm{F} \alpha}=2 k_{b} a^{2}$ \\
At large slip & $F_{x}=\mu F_{z} s_{x}\left(3 \theta-3\left(s_{x} \theta\right)^{2}+\left|s_{x} \theta\right|^{3}\right)$ & $F_{y}=\mu F_{z} s_{y}\left(3 \theta-3\left(s_{y} \theta\right)^{2}+\left|s_{y} \theta\right|^{3}\right)$ \\
& $1 / \theta=\frac{3 \mu F_{z}}{2 k_{b} a^{2}}$ & $1 / \theta=\frac{3 \mu F_{z}}{2 k_{b} a^{2}}$ \\
$\mid s_{\text {sliding }}$ & $x_{t 1}=\frac{4 k_{b} a^{3}}{3 \mu F_{z}} s_{x}-a$ & $x_{t 1}=\frac{4 k_{b} a^{3}}{3 \mu F_{z}} s_{y}-a$ \\
Break-away point &
\end{tabular}


Fig. 17 Equality of friction curves at pure slip types plotted against theoretical slip: (left) longitudinal and (right) lateral [23]
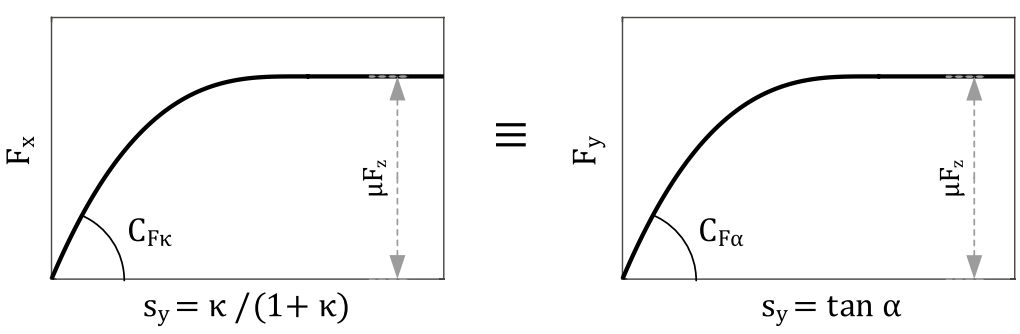

plotting the portions of the stationary and non-stationary regions vs. theoretical slips in Fig. 19. The ratios of the stationary to the non-stationary at equivalent theoretical slips for both $\alpha$ and $\kappa$ tests are presented in Fig. 20. For instance, at $5 \%$ slip, the stationary region in the $\alpha$ test is around $50 \%$ larger than for the $\kappa$ test.
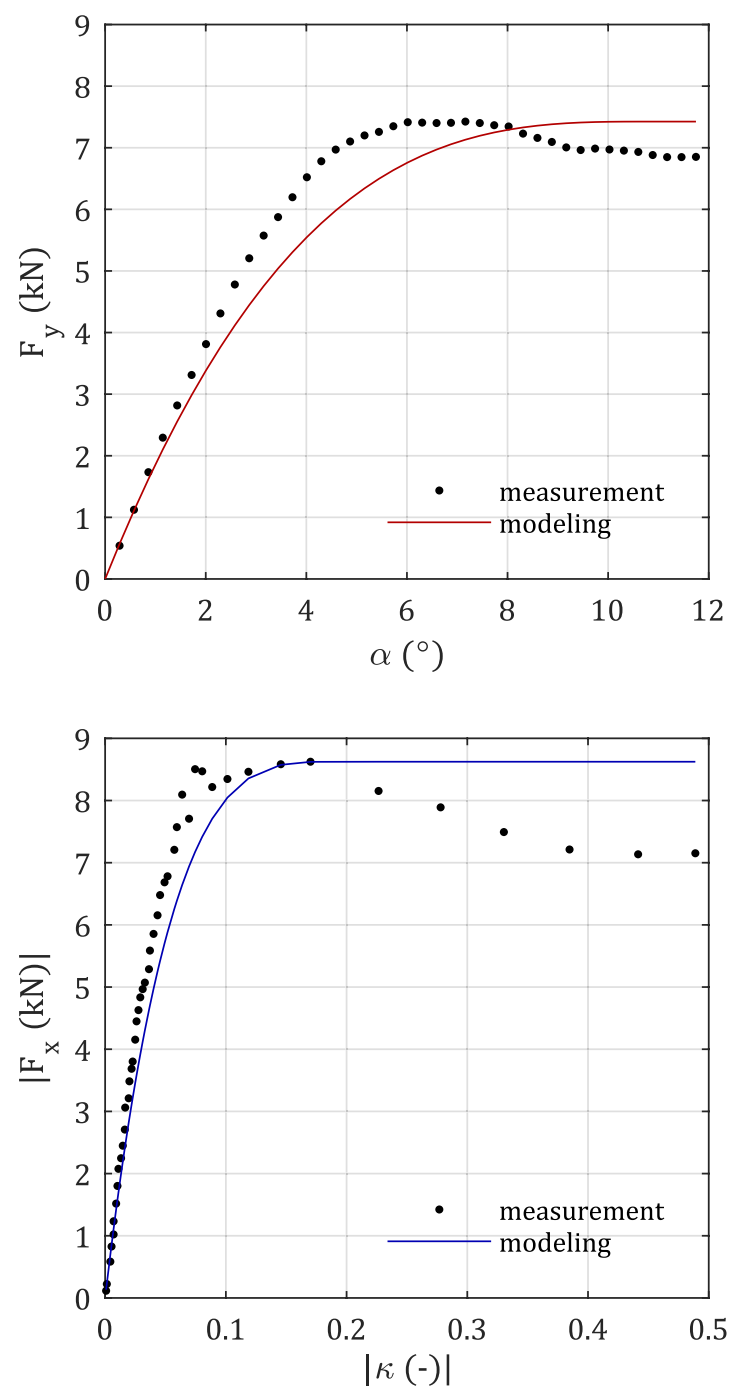

The theoretical slip as employed so far was solely a simple approach to demonstrate the basic but important underlying reason for no correlation between $\alpha$ and $\kappa$ sweep tests for tires. In reality, more factors play a role. When at equal theoretical slip, the frictional forces differ, it implicitly suggests that the slip velocities in the contact area differ. Referring back to the theoretical slip equations in Table 3 , these
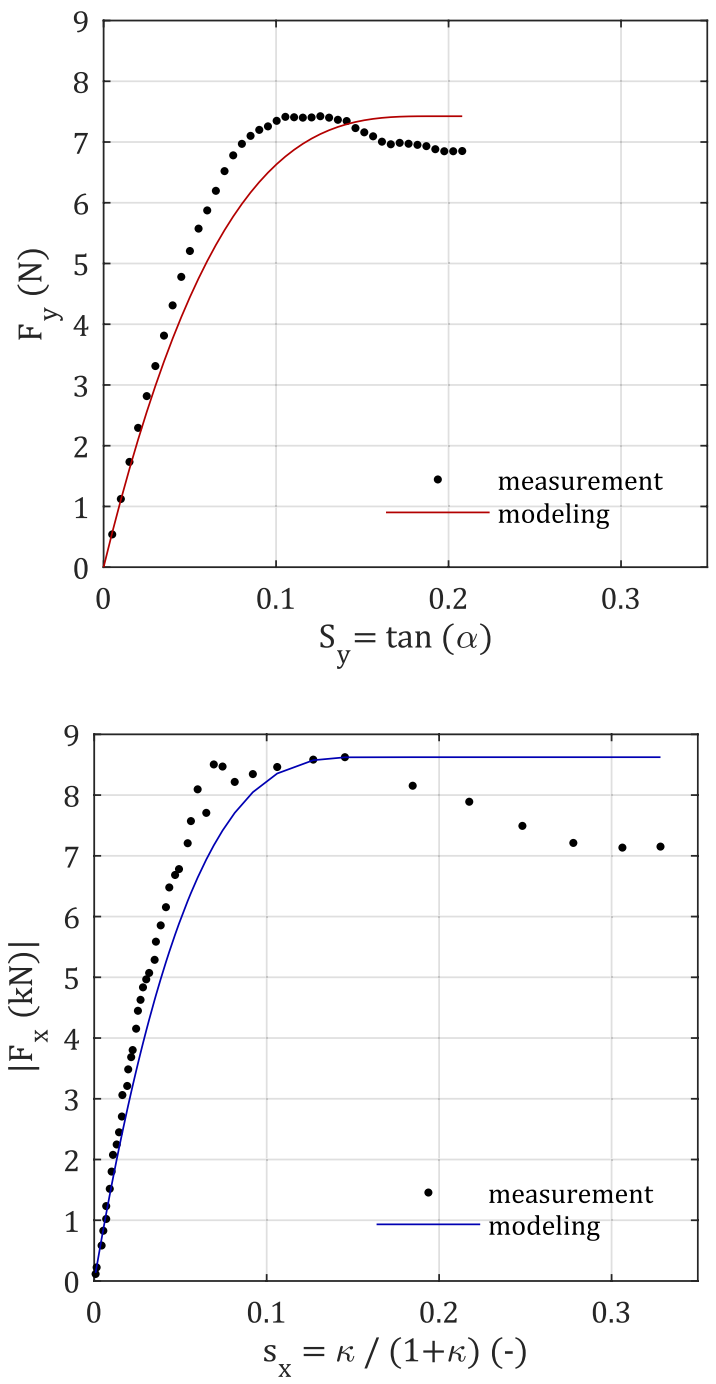

Fig. 18 Comparison of $\alpha$ and $\kappa$ tests at characteristic parameters and theoretical slips, first row: lateral sweep test, $F_{y}$ vs. $\alpha$ and $s_{y}$, second row: longitudinal sweep test, $F_{x}$ vs. $\kappa$ and $s_{x}$; for the same tread compound 
Fig. 19 Comparison of stationary and non-stationary regions at the theoretical slips for both $\alpha$ and $\kappa$ tests for the examples of Fig. 18

Fig. 20 Stationary/Non-stationary regions for $\alpha$ and $\kappa$ tests for the examples of Fig. 18
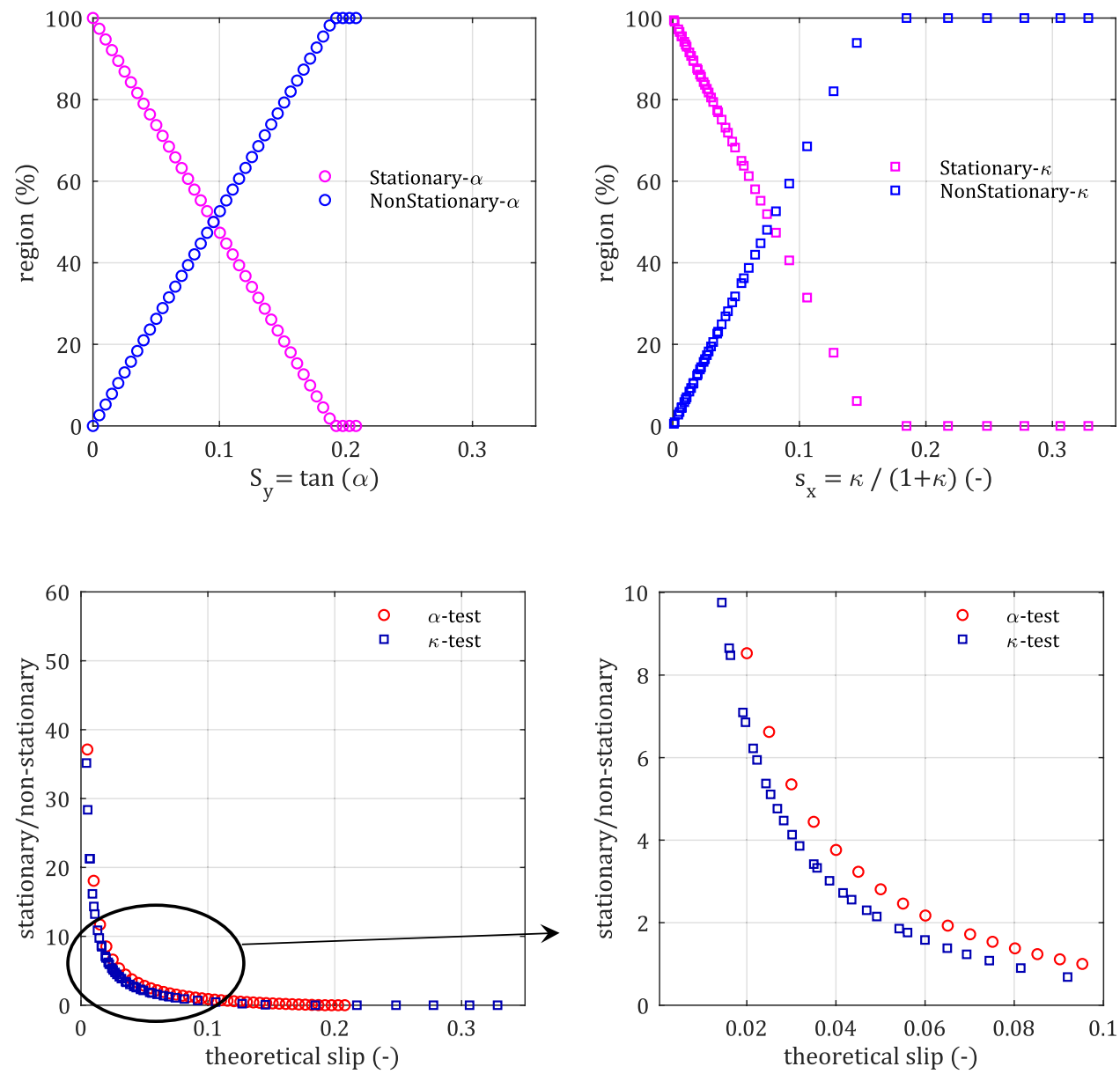

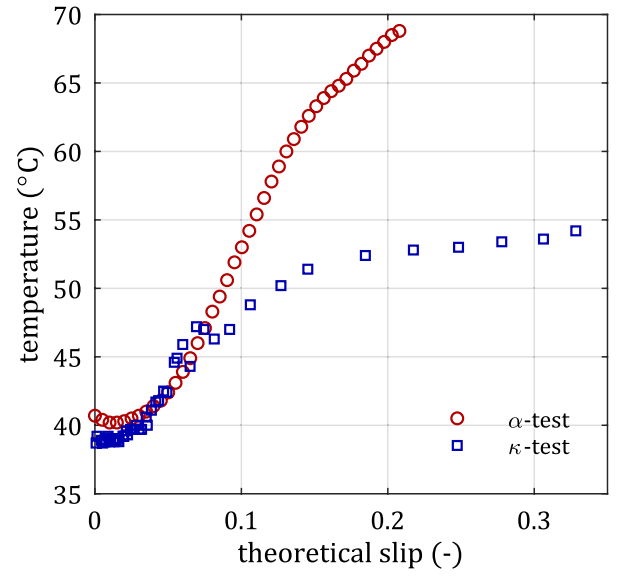

Fig. 21 Temperature profiles of $\alpha$ and $\kappa$ tests at equivalent theoretical slip for the examples of Fig. 18

defined measures do not imply equivalent slip velocities. For instance, at a theoretical slip of 0.1 , the slip velocities of the tire in $\alpha$ and $\kappa$ tests are $6 \mathrm{~km} / \mathrm{h}$ and $10 \mathrm{~km} / \mathrm{h}$, respectively. Furthermore, temperature and abrasion play a role. Figure 21 presents the temperature profiles of $\alpha$ and $\kappa$ tests at equivalent theoretical slip. Above 10\% slippage, the temperature difference becomes significant. This impacts the whole scenario of comparison.

Based on the current scenario, it was deduced so far that the $\alpha$ and $\mathrm{k}$ sweep tests of tires are not correlating theoretically and experimentally, and respectively, as the tire $\alpha$ data and LAT100 sweep measurements do correlate [1] mutatis mutandis, a correlation between tire $\kappa$ test and LAT100 sweep seems beyond the realm of possibilities for the acquired data.

\subsection{Comparison of $a$ Sweep Test of Grosch Wheel and Tire}

The possibility of the tire grip prediction for both $\alpha$ and $\kappa$ with the small rubber wheel is still highly attractive. If the underlying reasons for the correlation between LAT100 sweep and tire $\alpha$ test are fully comprehended, it opens the possibility to also mimic the situation for tire $\kappa$ test in a laboratory environment. Therefore, in the present section, the fundamental reasons for the correlations between the $\alpha$ sweep test of the Grosch wheel and tire are being further elaborated on. It benefits the subject in two ways: first, the 
correlations are not purely experimental by relying on test conditions, which makes it more reliable to acquire correlations with a new set of tire data; and second, the advantage of using a wheel-shaped specimen becomes more concrete to raise the possibility to also predict $\kappa$ sweep in a laboratory environment.

In the previous section, the comparison between tire $\alpha$ and $\kappa$ tests was elaborated in-depth. The same approach can now be employed for the $\alpha$ friction curves of the Grosch wheel and the tire at an equal theoretical measure of $s_{y}$. The similarity between the test modalities of lateral sweep of the tire and of the LAT100 seems to be the silver lining for the acquired correlations in the previous study [1]. Referring back to the acquired correlations, the slip angle sweep ranges up to the peak points in the measured friction curves $F_{y}$ vs. $\alpha$ of the LAT100 and the tire were different. The $\alpha$ friction curve of LAT100 approached a plateau roughly above $20^{\circ}$, which can be seen for example in Fig. 22 for the curves of $F_{y}$ vs. $\alpha$ or $s_{y}$ for compound C3 of our previous study [1] at a speed of $6.5 \mathrm{~km} / \mathrm{h}$ and a normal load of 55 N. Accordingly, Fig. 23 shows the stationary and non-stationary regions resulting from the modeling of the Grosch wheel. For the tire, the $\alpha$ friction curve reached full sliding at around $7^{\circ}$, see Fig. 18: $F_{y}$ vs. $\alpha$. The question is why the sweep ranges of the tire and LAT100 vary and more particularly with such a large difference, while the $\alpha$ test of the LAT100 and the tire did still correlate.

With the aid of the previously described model, it should be possible to reply to this question or at least provide some basic understanding. First, the beginning of the measured friction curve of the Grosch wheel shows a slight upward shift along the $y$-axis: see Fig. 22. This could implicitly indicate the presence of a pseudo-camber angle as mentioned in Sect. 4.2, which could be due to a non-uniformly vulcanized sample or an induced inclination angle owing to the path curvature. Second, due to this deviation, the $\alpha_{\text {sliding }}$ value for the Grosch wheel can be computed to be $30^{\circ}$ (or $s_{y}=0.53$ ), but the measurements suggested that full sliding occurred at $20^{\circ}$ (or $s_{y}=0.45$ ).

The correlations between the tire $\alpha$ and the LAT100 sweep tests were achieved for the cornering stiffness $C_{\mathrm{f}}$ and peak values of friction curves $F_{y}$ vs. $\alpha$. For $C_{f}$ and peak values, the dominant regions in the contact area are stationary and non-stationary, respectively. The correlation between the LAT100 and tire $\alpha$ tests for the $C_{\mathrm{f}}$ values are because of the
Fig. $22 \alpha$ sweep friction curve of the LAT100: $F_{y}$ vs. $\alpha$ or $s_{y}$ for the Grosch wheel at $6.5 \mathrm{~km} / \mathrm{h}$ and $55 \mathrm{~N}$ for compound $\mathrm{C} 3$
Fig. 23 Comparison of stationary and non-stationary regions at the theoretical slip $s_{y}$ for the Grosch wheel at $6.5 \mathrm{~km} / \mathrm{h}$ and $55 \mathrm{~N}$ for compound $\mathrm{C} 3$
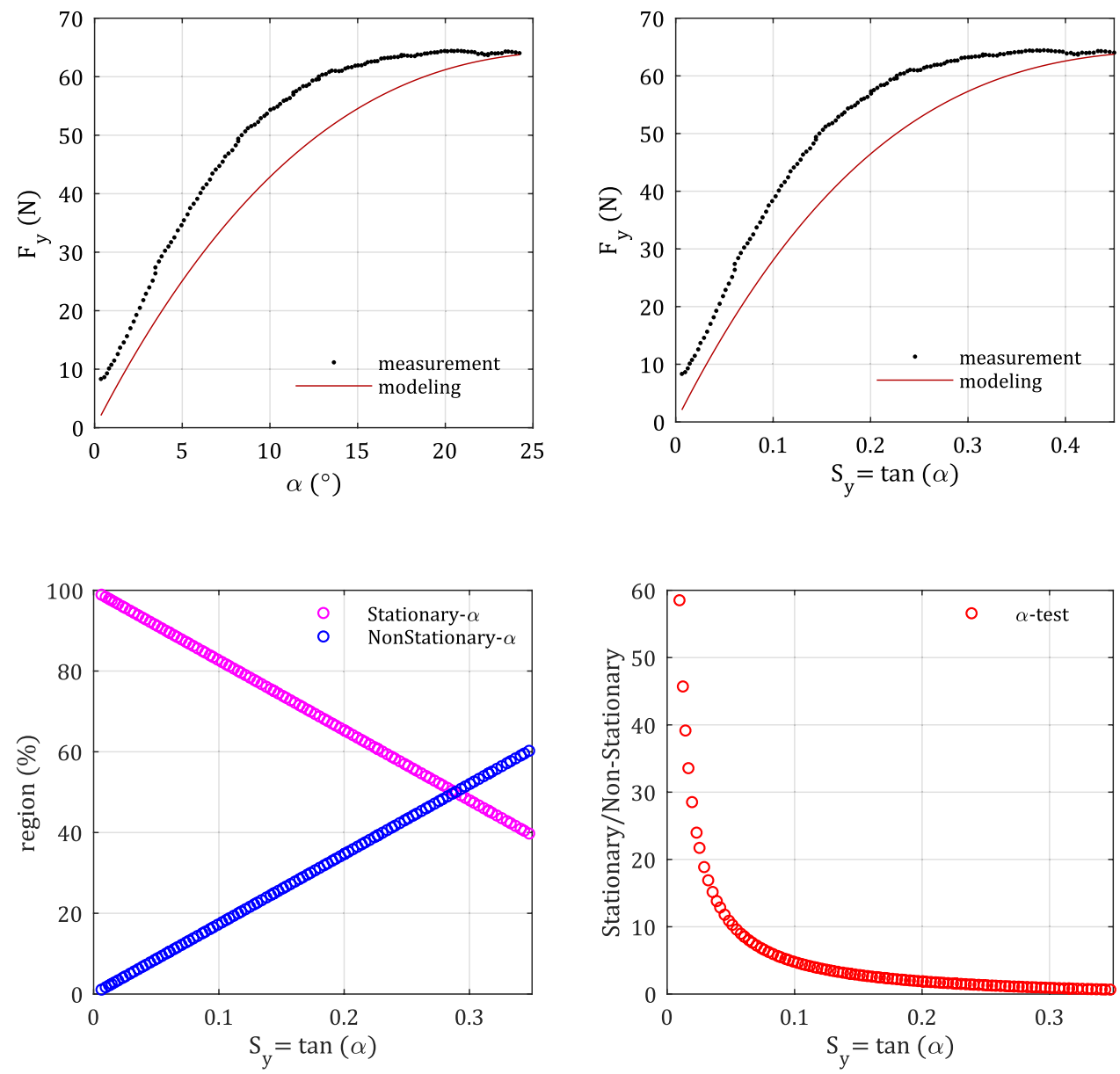
similarities between the friction mechanisms in the stationary regions of wheel samples, see Sect. 4.4. By recapping the comparison between the tire $\alpha$ and $\kappa$ tests, the ranking of the tread compounds for the stiffness parameters $C_{\mathrm{f} \alpha}$ and $C_{\mathrm{f} K}$ are comparable in order; suggesting that the tire tests of $\alpha$ and $\kappa$ in the primarily stationary regions correlate and have similar friction mechanisms, see Fig. 24.

The theoretical slip $s_{y}$ values of c.a. 0.17 and 0.53 for the tire and Grosch wheel do differ at the peak or $\alpha_{\text {sliding }}$ points that indicate that both samples are in the non-stationary region; it theoretically implies that no correlation can occur! Despite that, the $\alpha$ tests of LAT100 and tire experimentally did correlate at the peak values. This paradox can be solved by comparison of the slip velocities of the LAT100 Grosch wheel and the corresponding tire. The slip velocity in lateral test modality of the tire is defined as follows:

$-V_{s y}=V_{x} \tan \alpha$.

Based on this equation and the tire data, at $60 \mathrm{~km} / \mathrm{h}$ velocity of travel and theoretical slip of 0.1 , the tire slip velocity is around $5.8 \mathrm{~km} / \mathrm{h}$ in the lateral direction. The velocity of the Grosch wheel which delivers the correlation with the tire data was $6.5 \mathrm{~km} / \mathrm{h}$. This speed was eight times smaller than the tire speed as shown in the previous study [1]. Therefore, the measurement at lower velocity is compensated with sweeping to a larger range of slip angles to maintain a level of slip velocity that can induce full sliding or a full non-stationary region for the Grosch wheel. At an equivalent slip velocity of $5.8 \mathrm{~km} / \mathrm{h}$ for the Grosch wheel, the slip angle for the traveling speed of $6.5 \mathrm{~km} / \mathrm{h}$ can be calculated according to Eq. (34) to be $41^{\circ}$. This shows why LAT100 peaks at larger slip angles. It should be noted that in the measurements, full sliding begins at around $25^{\circ}$ slip angle. This discrepancy is due to the assumptions of the brush model. It is noteworthy that the modeling was done under numerous assumptions. The contact width of the Grosch wheel, $2 b$ is larger than the contact length $2 a$; an implementation of multi-rows of bristles is more realistic and can provide more precise information regarding the deformation of the Grosch wheel. Furthermore, the curvature of the disk countersurface path should be taken into account. The counter-surface disk curvature creates a distribution of slip velocities within the contact patch of the Grosch wheel as a function of $\alpha$ and $r$, the radius of an arbitrary point in the contact area to the center of the counter-surface disk, see Fig. 2 and Eq. (3). The range of influence of the disk curvature on the variation of contact patch radius and slip velocity can be approximately computed for the Grosch wheel according to Sect. 2.2, as shown in Fig. 25; the
Fig. 24 Ranking of tire $\alpha$ and $\kappa$ sweep tests at small slip for all compounds of the previous manuscript [1]
Fig. 25 Influence of the path curvature on the variation of the radius of contact points to the center of disk counter-surface and slip velocity, at disk traveling speed $6.5 \mathrm{~km} / \mathrm{h}$ and slip angle $25^{\circ} ; 2 a$ Grosch contact length, $2 b$ : contact width
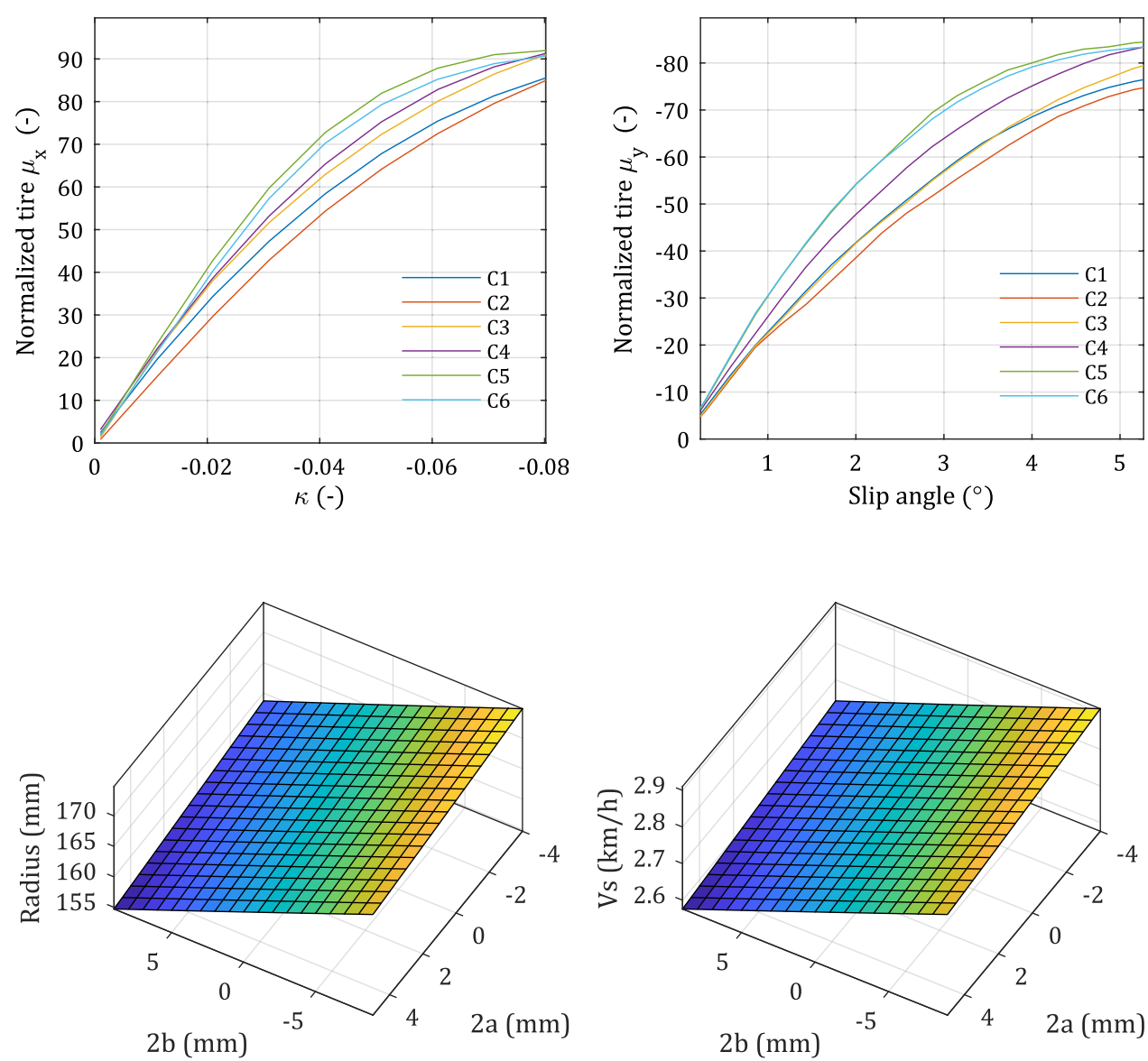
farther the contact point from the center of the counter-surface disk, the larger the slip velocity. The difference between the highest and the lowest slip velocities $\Delta V_{\mathrm{s}}$ in the contact area is influenced by input parameters of $\alpha$ and disk traveling velocity, see Fig. 26. The $\Delta V_{\mathrm{s}}$ can even reach $10 \mathrm{~km} / \mathrm{h}$ at a very high speed and slip angle. It should be noted that the deflection of rubber with the sweep is disregarded in this calculation.

Hence, it is possible to highlight the underlying reasons for the obtained correlations between the Grosch wheel and tire by comparison of the slip velocities and normal load ranges and friction mechanisms concerning the slippage regions. And if these assumptions are correct, it should be possible to predict $\kappa$ sweep with a small solid rubber wheel just by choosing the right test configuration and conditions. This could be obtained even though the experimental samples of the Grosch wheel were compared with tires with $25 \%$ contribution of the carcass construction and tread pattern (see Fig. 4) for friction and the counter-surfaces being corundum-based vs. asphalt.

Last but not least, the radial thickness of around $25 \mathrm{~mm}$ for the LAT100 sample should be reduced; thus, the implementation of the brush model with the assumption of a rigid carcass for the Grosch wheel would be more realistic. This could be further optimized to be able to exploit the LAT100 as a versatile predictor for tire friction test modalities.

\section{Conclusions}

By using the renowned physical 'brush model', the dynamics and physics of the Grosch rubber wheel on the LAT100 countersurface as tribometer have been modeled. The behavior in lateral and longitudinal movements, similar to a real tire, were investigated. The differences between the $\alpha$ and $\kappa$ sweep test modalities have been explained at a defined equivalent measure called theoretical slip. It was deduced that the type of test modality leads to different friction mechanisms in the contact patch even at similar test conditions. The underlying reasons for the correlation

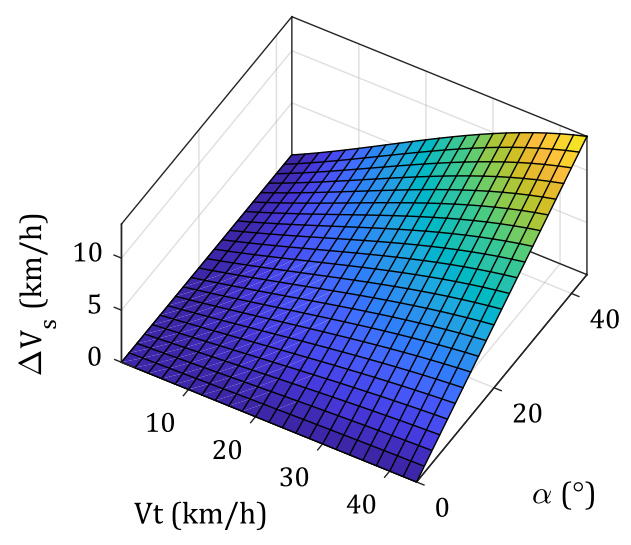

Fig. 26 The $\Delta V_{\mathrm{s}}$ between the highest and the lowest slip velocities in the contact area vs. disk traveling speed and slip angle between LAT100 and tire $\alpha$ sweep tests have been highlighted. The importance of a laboratory wheel sample geometry compared to a pure sliding body specimen could be substantiated by recognizing the two regions: stationary and non-stationary in the contact area similar to the tire. This resulted in similar friction mechanisms for the Grosch wheel as for a rolling tire. In reality in ordinary driving, a passenger car tire on the road operates in a slip angle range between $2^{\circ}$ and $6^{\circ}$, while the peak of the friction curve typically occurs at $4^{\circ}-7^{\circ}$ of slip angles. This explains the importance of friction curves studied with the Grosch wheel because it provides insight into the stationary friction.

It has been deduced that the $\alpha$ and $\kappa$ sweep tests of tires are not correlating theoretically nor experimentally, and as the tire $\alpha$ data and LAT100 sweep measurements do correlate, a correlation between tire $\kappa$ tests and LAT100 sweep data seems beyond the realm of possibilities. However, the present study does provide an indication that it should be possible to predict $\kappa$ sweep as well with a small solid rubber wheel, by choosing the right test configuration and modality.

The present study also suggests that modifications of the Grosch wheel could enhance the predictability of tire friction on the road, such as by improvement of the geometry of the specimen. The radial thickness and width of the Grosch wheel can be optimized by utilizing a multi-row brush model in which the assumptions are better tailored to reality. Therefore, by using such an optimized small rubber wheel, it should be possible to predict tire grip in the laboratory for material development which saves cost and time rather than testing tread compounds by building full-scale tires.

\section{Appendix}

See Table 4.

Table 4 The physical, mechanical, and DMTA properties of the tread compounds $\mathrm{A}$ and $\mathrm{E}$

\begin{tabular}{lll}
\hline Properties & Comp A & Comp E \\
\hline Hardness ( $\left.{ }^{\circ} \mathrm{Sh} \mathrm{A}\right)$ & 71 & 61 \\
Tensile strength (MPa) & 15.7 & 17.7 \\
Elongation at break (\%) & 500 & 585 \\
$G^{\prime}(\mathrm{MPa})$ at $10 \mathrm{~Hz}, 40{ }^{\circ} \mathrm{C}, \mathrm{S} 6 \%^{\mathrm{a}}$ & 2.3 & 1.6 \\
$G^{\prime}(\mathrm{MPa})$ at $10 \mathrm{~Hz}, 40^{\circ} \mathrm{C}, \mathrm{S} 0.1 \%^{\mathrm{b}}$ & 13.8 & 5.4 \\
$G^{\prime}(\mathrm{MPa})$ at $10 \mathrm{~Hz}, 40^{\circ} \mathrm{C}, \mathrm{S} 0.1 \%^{\mathrm{c}}$ & 7.8 & 3.7 \\
$G^{\prime}(\mathrm{MPa})$ at $1 \mathrm{~Hz}, 40^{\circ} \mathrm{C}, \mathrm{SO} .1 \%^{\mathrm{c}}$ & $7.6^{*}$ & 3.1 \\
$G^{\prime}(\mathrm{MPa})$ at $10 \mathrm{~Hz}, 40^{\circ} \mathrm{C}, \mathrm{S} 6 \%^{\mathrm{c}}$ & 3.1 & 2.3 \\
$G^{\prime}(\mathrm{MPa})$ at $1 \mathrm{~Hz}, 40^{\circ} \mathrm{C}, \mathrm{S} 6 \%^{\mathrm{c}}$ & 2.9 & 2.0 \\
\hline
\end{tabular}

*The italic row is used for the modeling calculations

${ }^{\mathrm{a}}$ The data are taken from a temperature sweep measurement

${ }^{\mathrm{b}}$ The data are taken from a strain sweep measurement

${ }^{\mathrm{c}}$ The data are taken from a frequency sweep measurement 
Fig. 27 An arbitrary point A with distance $R_{\mathrm{A}}$ from the center of the disk in the contact area of the Grosch wheel

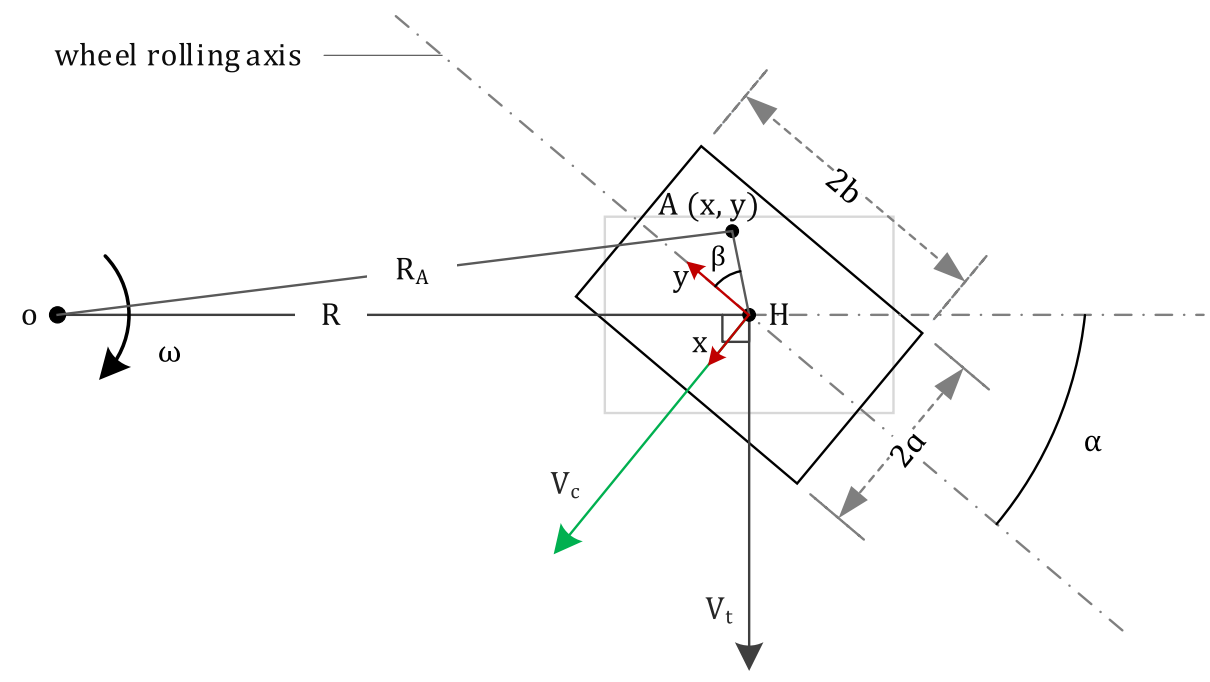

Derivation of Eq. (3) based on Fig. 27:

based on cosine law on the oblique triangle of $(\mathrm{OAH})$ :

$R_{A}^{2}=R^{2}+|A H|^{2}-2 R|A H| \cos (\alpha+\beta)$

The magnitude of AH based on the coordinate of A point can be defined as follows:

$|A H|^{2}=x^{2}+y^{2}$

Based on the compound angle formula of $\cos (\alpha+\beta)$ and by substituting Eqs. (36) in (35) and definition of sin and cos based on the coordinate of point $A, r$ can be derived as follows:

$r(x, y, \alpha)=R^{2}+x^{2}+y^{2}-2 R(y \cos \alpha+x \sin \alpha)$.

The deformation of rubber in the contact area is not considered.

Acknowledgements The present research was financed by Apollo Tyres Global R\&D B.V., Enschede, the Netherlands. The authors would like to express their gratitude for the support.

Open Access This article is licensed under a Creative Commons Attribution 4.0 International License, which permits use, sharing, adaptation, distribution and reproduction in any medium or format, as long as you give appropriate credit to the original author(s) and the source, provide a link to the Creative Commons licence, and indicate if changes were made. The images or other third party material in this article are included in the article's Creative Commons licence, unless indicated otherwise in a credit line to the material. If material is not included in the article's Creative Commons licence and your intended use is not permitted by statutory regulation or exceeds the permitted use, you will need to obtain permission directly from the copyright holder. To view a copy of this licence, visit http://creativecommons.org/licenses/by/4.0/.

\section{References}

1. Salehi, M., et al.: A new horizon for evaluating tire grip within a laboratory environment. Tribol. Lett. 68(1), 37 (2020)
2. Gutzeit, F., Kröger, M.: Experimental and theoretical investigations on the dynamic contact behavior of rolling rubber wheels. In: Besdo, D., et al. (eds.) Elastomere Friction: Theory, Experiment and Simulation, pp. 221-249. Springer, Berlin (2010)

3. Steen, R.V.D., Lopez, I., Nijmeijer, H.: Experimental and numerical study of friction and giffness characteristics of small rolling tires. Tire Sci. Technol. 39(1), 5-19 (2011)

4. Dorsch, V., Becker, A., Vossen, L.: Enhanced rubber friction model for finite element simulations of rolling tyres. Plast. Rubber Compos. 31(10), 458-464 (2002)

5. Salehi, M., et al.: Measuring rubber friction using a Laboratory Abrasion Tester (LAT100) to predict car tire dry ABS braking. Tribol. Int. 131, 191-199 (2019)

6. Nguyen, V.H., et al.: An advanced abrasion model for tire wear. Wear 396-397, 75-85 (2018)

7. Wu, J., et al.: Wear predicted model of tread rubber based on experimental and numerical method. Exp. Tech. 42(2), 191-198 (2018)

8. Salehi, M., et al.: Vorhersagen für das Bremsverhalten von Reifen durch Bestimmung der Gummireibung. Gummi Fasern Kunststoffe 72, 24-33 (2019)

9. Grosch, K.A.: Correlation between road wear of tires and computer road wear simulation using laboratory abrasion data. Rubber Chem. Technol. 77(5), 791-814 (2004)

10. Grosch, K.A.: Goodyear medalist lecture. Rubber friction and its relation to tire traction. Rubber Chem. Technol. 80(3), 379-411 (2007)

11. Salehi, M.: LAT100, Prediction of Tire Dry Grip. University of Twente, Enschede: Ipskamp Printing (2017)

12. Grosch, K.A.: Rubber abrasion and tire wear. Rubber Chem. Technol. 81(3), 470-505 (2008)

13. Salehi, M.: Prediction of Tire Grip: A New Method for Measurement of Rubber Friction Under Laboratory Conditions. University of Twente, Twente (2020)

14. Heinz, M., Grosch, K.A.: A laboratory method to comprehensively evaluate abrasion, traction and rolling resistance of tire tread compounds. Rubber Chem. Technol. 80(4), 580-607 (2007)

15. Heinz, M.: A laboratory abrasion testing method for use in the development of filler systems. In: Technical Report Rubber Reinforcement Systems. Evonik Industries GmbH, Essen (2015)

16. Grosch, K.A.: A new way to evaluate traction-and wear properties of tire tread compounds. In: Rubber Division. American Chemical Society, Cleveland (1997)

17. Heinz, M.: A universal method to predict wet traction behaviour of tyre tread compounds in the laboratory. J. Rubber Res. 13(2), 91-102 (2010) 
18. Salehi, M., et al.: Parameter optimization for a laboratory friction tester to predict tire ABS braking distance using design of experiments. Mater. Des. 194, 108879 (2020)

19. Miller, S.L. et al.: Calculation longitudinal wheel slip and tire parameters using GPS velocity. In: The American Control Conference. Arlington (2001)

20. New Tires vs Worn Tires - What Performs Best?, in Engineering Explained June 2018.

21. The Tyre Grip: Société de Technologie Michelin., clermont-ferrand, France (2001)

22. Besselink, I.J.M.: Tire characteristics and modeling. In: Lugner, P. (ed.) Vehicle Dynamics of Modern Passenger Cars, pp. 47-108. Springer International Publishing, Cham (2019)

23. Pacejka, H.B.: Chapter 3-theory of steady-state slip force and moment generation. In: Tyre and Vehicle Dynamics, 2nd edn., pp. 90-155. Butterworth-Heinemann, Oxford (2006)

24. Braghin, F., et al.: Tyre wear model: validation and sensitivity analysis. Meccanica 41(2), 143-156 (2006)

25. Kane, M., Cerezo, V.: A contribution to tire/road friction modeling: from a simplified dynamic frictional contact model to a "Dynamic Friction Tester" model. Wear 342-343, 163-171 (2015)

26. Schramm, D., Hiller, M., Bardini, R.: Modeling of the roadtire-contact. In: Vehicle Dynamics: Modeling and Simulation. Springer Berlin, pp. 145-186 (2018)

27. Erdogan, G.: Tire Modeling, Lateral and Longitudinal Tire Forces. University of Minnesota, Minneapolis (2009)

28. Ossama Mokhiamar, M.A.: Simultaneous optimal distribution of lateral and longitudinal tire forces for the model following control. ASME 126, 753-763 (2004)
29. Walloch, F.: The impact of tire design on vehicle braking performance. In: SAE World Conference. Continental Tire (2008)

30. Jacob Svendenius, B.W.: Review of wheel modeling and friction estimation. In: Department of Automatic Control. Lund institute of technology (2003)

31. Schallamach, A., Turner, D.M.: The wear of slipping wheels. Wear 3(1), 1-25 (1960)

32. Grosch, K.A.: Rubber friction and tire traction. In: The Pneumatic Tire. NHTSA, pp. 421-473 (2006)

33. Rosu, I., et al.: Experimental and numerical simulation of the dynamic frictional contact between an aircraft tire rubber and a rough surface. Lubricants 4(3), 29 (2016)

34. Ray, L.R.: Nonlinear tire force estimation and road friction identification: simulation and experiments 1,2. Automatica 33(10), 1819-1833 (1997)

35. Pacejka, H.B.: In Tyre and Vehicle Dynamics, 2 edn. ButterworthHeinemann, Oxford, pp. 613-628 (2006)

36. Tiwari, A., et al.: Rubber contact mechanics: adhesion, friction and leakage of seals. Soft Matter 13(48), 9103-9121 (2017)

37. Tolpekina, T.V., Persson, B.N.J.: Adhesion and friction for three tire tread compounds. Lubricants 7(3), 20 (2019)

38. Pacejka, H.B.: Chapter 1-tyre characteristics and vehicle handling and stability. In: Tyre and Vehicle Dynamics, 2nd edn., pp. 1-60. Butterworth-Heinemann, Oxford (2006)

Publisher's Note Springer Nature remains neutral with regard to jurisdictional claims in published maps and institutional affiliations. 